\title{
1 ACE2 Pathway Regulates Thermogenesis and Energy
}

2 Metabolism

3

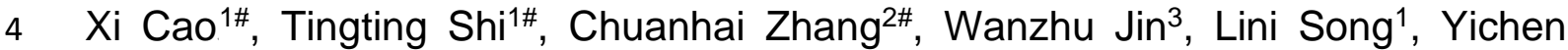
5 Zhang ${ }^{1}$, Jingyi Liu ${ }^{1}$, Fangyuan Yang ${ }^{1}$, Charles $\mathrm{N}$ Rotimi ${ }^{4}$, Amin $\mathrm{Xu}^{5}$ and Jinkui 6 Yang $^{1} \bowtie$

$7 \quad{ }^{1}$ Beijing Diabetes Institute, Beijing Key Laboratory of Diabetes Research and 8 Care, Beijing Tongren Hospital, Capital Medical University, Beijing 10730, China

92 Beijing Advanced Innovation Center for Food Nutrition and Human Health, 10 College of Food Science and Nutritional Engineering, China Agricultural 11 University, Beijing, 100083, China

${ }^{3}$ Key Laboratory of Animal Ecology and Conservation Biology, Institute of Zoology, and State Key Laboratory of Brain and Cognitive Sciences, Institute of Biophysics, Chinese Academy of Sciences, Beijing, China

${ }^{4}$ Center for Research on Genomics and Global Health, National Human Genome Research Institute, National Institutes of Health, Bethesda, Maryland, USA

${ }^{5}$ State Key Laboratory of Pharmaceutical Biotechnology, Department of Medicine, University of Hong Kong, Hong Kong, China

\# These authors contribute equally to this paper.

$\triangle$ Address correspondence and reprint requests to Professor JinKui Yang, Department of Endocrinology, Beijing Tongren Hospital, Capital Medical University, Beijing 100730, China

Tel: +86-10-58268445

Fax: $+86-10-65288736$

Cell: $+86-13911167636$

E-mail: jkyang@ccmu.edu.cn 


\section{Abstract}

2 Identification of key regulators of energy homeostasis holds important therapeutic

3 promise for metabolic disorders, such as obesity and diabetes. ACE2 cleaves

4 angiotensin II (Ang II) to generate Ang-(1-7) which acts mainly through the Mas

5 receptor. Here, we identify ACE2 pathway as a critical regulator in the

6 maintenance of thermogenesis and energy expenditure. We found that ACE2 is

7 highly expressed in brown adipose tissue (BAT) and that cold stimulation

8 increases ACE2 and Ang-(1-7) levels in BAT and serum. ACE2 knockout mice

9 (ACE2 $\left.2^{-/ y}\right)$, Mas knockout mice $\left(\right.$ Mas $\left.^{-/}\right)$, and the mice transplanted with brown

10 adipose tissue from $\mathrm{Mas}^{-/}$mice displayed impaired thermogenesis. In contrast, impaired thermogenesis of $\mathrm{db} / \mathrm{db}$ obese diabetic mice and high-fat diet-induced obese mice were ameliorated by overexpression of ACE2 or continuous infusion of Ang-(1-7). Activation of ACE2 pathway was associated with improvement of metabolic parameters, including blood glucose, lipids and energy expenditure in multiple animal models. Consistently, ACE2 pathway remarkably enhanced the browning of white adipose tissue. Mechanistically, we showed that ACE2 pathway activated Akt/FoxO1 and PKA pathway, leading to induction of UCP1 and activation of mitochondrial function. Our data propose that adaptive thermogenesis requires regulation of ACE2 pathway and highlight novel therapeutic targets for the treatment of metabolic disorders.

Key Words: ACE2; Angiotensin-(1-7); obesity; diabetes; brown adipose tissue; thermogenesis 


\section{3 / 37}

\section{Introduction}

2 Energy imbalance and the associated metabolic syndromes have become a

3 worldwide public health problem. Thus, identifying factors that can stimulate

4 energy expenditure is instrumental to the development of therapeutics to reduce

5 obesity associated disorders that affect over $10 \%$ of the world population (Dong,

6 Lin, Lim, Jin, \& Lee, 2017). In the renin-angiotensin system (RAS),

7 angiotensin-converting enzyme 2 (ACE2) cleaves angiotensin II (Ang II) to 8 generate angiotensin-(1-7) (Ang-(1-7)). Ang-(1-7) is a heptapeptide hormone

9 which acts mainly through G-protein-coupled receptor Mas (Santos et al., 2003). ACE2-Ang-(1-7)-Mas pathway works as a negative regulator of ACE-Ang II pathway in multiple disease states (Clarke \& Turner, 2012).

In this study we reported the effects of ACE2 pathway on regulating thermogenesis and energy metabolism via modulating mitochondrial function. We found that ACE2 knockout $\left(A C E 2^{-/ y}\right)$ and Mas knockout $\left(\mathrm{Mas}^{-/}\right)$mice are cold intolerance. We provided compelling genetic, metabolic, physiological, histological, cellular, and molecular evidence to demonstrate that ACE2 pathway is a critical regulator in the maintenance of energy expenditure. This pathway regulates function of brown adipose tissue (BAT) and systemic energy metabolism. Mechanistically, ACE2 pathway activates both Akt/FoxO1 signaling and PKA signaling, leading to induction of uncoupling protein-1 (UCP1) and activation of mitochondrial function. Therefore, ACE2 pathway is a potential treatment target for metabolic disorders including diabetes, obesity, and even cardiovascular diseases. 


\section{4 / 37}

1 Acute cold exposure increases components of ACE2 pathway

2 The major tissue of the body where energy is converted into the form of heat to

3 maintain the body temperature is BAT. We found both mRNA level and protein

4 level of ACE2 and Mas in BAT were obviously higher than the ones in

5 subcutaneous white adipose tissue (scWAT) and epididymal white adipose tissue

6 (eWAT) in mice (Figure 1A and B). Acute cold exposure caused a significant

7 up-regulation of ACE2 protein expression in BAT (Figure 1C). Meanwhile, ACE2

8 mRNA levels in BAT, scWAT and eWAT, and Mas mRNA levels in BAT and eWAT also increased after exposed to $4^{\circ} \mathrm{C}$ for 48 hours (Figure 1D and E). ACE2 and Ang-(1-7) were also marginally increased in serum upon cold challenge (Figure $1 F$ and $G$ ). These results demonstrated a selective induction of ACE2 pathway in thermogenic adipose depots (BAT and scWAT) and blood circulation in response to cold environment.

ACE2 promotes thermogenesis and energy metabolism 


\section{5 / 37}

1 comprehensive laboratory animal monitoring system (CLAMS). We observed a

2 decreased oxygen consumption $\left(\mathrm{VO}_{2}\right)$, carbon dioxide release $\left(\mathrm{VCO}_{2}\right)$ and

3 energy expenditure (EE) in $A C E 2^{-1 y}$ mice (Figure $2 C, D$ and $E$ ), without

observable changes in food and/or water intake as well as physical activity, compared to the WT mice (Figure 2-figure supplement 1D-F).

To further examine the differences in energy expenditure among these animals, we performed a cold tolerance test in order to gauge adaptive thermogenesis. $A C E 2^{-1 y}$ mice had lower thermogenesis than the WT mice in a cold environment $\left(4^{\circ} \mathrm{C}\right)$ (Figure 2-figure supplement 1G). To explore the source of thermogenesis, we analyzed the non-shivering thermogenesis (NST) of ACE2 KO mice in thermoneutral condition $\left(30^{\circ} \mathrm{C}\right)$, ambient temperature $\left(22^{\circ} \mathrm{C}\right)$ and acute cold $\left(4^{\circ} \mathrm{C}\right)$ for 8 hours. $A C E 2^{-/ y}$ mice had lower thermogenesis than the WT mice in either $22^{\circ} \mathrm{C}$ or $4^{\circ} \mathrm{C}$ (Figure $\left.2 F\right)$. This temperature difference was monitored by an infrared camera (Figure 2G).

To investigate whether ACE2 induced thermogenesis was related to BAT function, we performed the Positron emission tomography-computed tomography (PET-CT) analysis and the results showed a higher PET-CT signal in BAT of the HFD-induced WT mice than ACE2/y mice (Figure 2H). As expected, BAT in $A C E 2^{1 / y}$ mice displayed larger lipid droplets but reduced multilocular structures compared to the WT mice, and had reduced UCP1 expression (Figure 21).

To evaluate the significance of cold-induced ACE2 for thermogenic function of BAT, the expression levels of a network of genes and proteins controlling energy expenditure and thermogenic programming were measured. Protein levels (UCP1, PGC-1 $\alpha$ and ATP5A) (Figure 2J) and mRNA levels (UCP1, PRMD16 and PPARY) (Figure 2-figure supplement $1 \mathrm{H}$ ) in BAT from $A C E 2^{/ y}$ were obviously decreased. 


\section{6 / 37}

To validate the above-mentioned change of thermogenesis of BAT was cell autonomous, primary brown adipocytes from $A C E 2^{-1 y}$ mice was fractionated and differentiated in vitro. Notably, the protein and mRNA expression of known BAT markers were robustly decreased in ACE2 deficient primary brown adipocytes (Figure 2K, Figure 2-figure supplement 1). Immunohistochemistry was applied to study the level of UCP1 in primary brown adipocytes differentiated from the BAT of the $A C E 2^{-1 y}$ mice. The result showed the UCP1 expression was reduced in the ACE2 deficiency primary brown adipocytes (Figure 2L). More importantly, the oxygen consumption rate (OCR) was significantly decreased in ACE2 deficient primary brown adipocytes (Figure 2M).

As a complementary approach to the $\mathrm{KO}$ mouse models, we carried out gain-of-function studies using ACE2 over expression in obese diabetic $\mathrm{db} / \mathrm{db}$ mice. One week following adenovirus induced ACE2 over-expression (Ad-ACE2) by tail vein injection in the $\mathrm{db} / \mathrm{db}$ mice, both ACE2 (Figure 3-figure supplement 2A) in BAT and circulating Ang-(1-7) (Figure 3A) were increased. Consistent with our previous study, the Ad-ACE2-treated mice exhibited an improved metabolic profile as indicated by the significant alleviation of glucose intolerance (Figure 3-figure supplement 2B). Notably, although no observable change on the body weight was observed in the two groups (Figure 3B), serum triglyceride levels decreased in the Ad-ACE2-treated mice (Figure 3-figure supplement 2C), as well as a minor change in serum cholesterol levels (Figure 3-figure supplement 2D).

Notably, the Ad-ACE2 treated $\mathrm{db} / \mathrm{db}$ mice had increased energy expenditure $\left(\mathrm{VO}_{2}, \mathrm{VCO}_{2}\right.$ and EE) (Figure $\left.3 \mathrm{C}-\mathrm{E}\right)$. There was no obvious change in food and/or water intake as well as physical activity (Figure 3-figure supplement 2E-G). 


\section{7 / 37}

1 We measured rectal temperature and infrared thermal imaging in the $\mathrm{db} / \mathrm{db}$

2 mice that BAT activity was defective as same as the ones in previous

3 observations (Trayhurn \& Wusteman, 1990; Z. Zhang et al., 2014). The results showed that the thermogenesis of the $\mathrm{db} / \mathrm{db}$ mice was severely impaired (Figure 3-figure supplement $2 \mathrm{H}, \mathrm{I}$. As expected, the Ad-ACE2 treated db/db mice exhibited better thermogenesis than the control mice in ambient temperature $\left(22^{\circ} \mathrm{C}\right)$ and acute cold $\left(4^{\circ} \mathrm{C}\right)$ conditions (Figure 3F, G, Figure 3-figure supplement 2J). Accordingly, PET-CT result showed that BAT was activated in the Ad-ACE2 treated db/db mice (Figure 3H). Moreover, BAT in the Ad-ACE2 treated db/db mice had smaller lipid droplets but increased multi-locular structures, and had increased UCP1 expression compared with the control group (Figure 3I). The protein levels of UCP1, ATP5A and UQCRC2 were significantly increased in the BAT from the Ad-ACE2 treated mice (Figure 3J). Consistently, the mRNA levels, including UCP1, ATPsyn, COX8b, COX7a, AP2, CPT-1a, PRDM16, $P G C-1 \alpha$ and $P G C-1 \beta$, were increased in the BAT from the Ad-ACE2 treated $\mathrm{db} / \mathrm{db}$ mice (Figure 3K). Taken together, these results indicated that ACE2 effectively regulated the mitochondrial biogenesis and respiratory function in brown adipocytes.

\section{Ang-(1-7) promotes thermogenesis and energy metabolism}

To explore the direct physiological roles of Ang-(1-7) in cold-induced adaptive thermogenesis, Ang-(1-7) administration by subcutaneous implantation of micro-osmotic pumps in the $\mathrm{db} / \mathrm{db}$ and the HFD-induced obese mice were employed. Serum Ang-(1-7) was increased in Ang-(1-7) treated mice (Figure 4-figure supplement 3A). There are no significant differences in body weight between the Ang-(1-7) treated $\mathrm{db} / \mathrm{db}$ mice and the $\mathrm{db} / \mathrm{db}$ control mice (Figure $4 A$ ), however, Ang-(1-7) treated db/db mice has an improved glucose tolerance ability 


\section{$8 / 37$}

1 (Figure 4-figure supplement 3B) and better lipid profiles (Figure 4-figure

2 supplement $3 C, D)$. The Ang-(1-7) treated HFD-induced obese mice displayed a

3 lower body weight compared to the control (Figure 4B).

Notably, the Ang-(1-7) treated db/db mice had increased energy expenditure $\left(\mathrm{VO}_{2}, \mathrm{VCO}_{2}\right.$ and $\mathrm{EE}$ ) (Figure $\left.4 \mathrm{C}-\mathrm{E}\right)$ without any changes in food and/or water intake as well as physical activity (Figure 4-figure supplement 3E-G). Moreover, the Ang-(1-7) treated $\mathrm{db} / \mathrm{db}$ and the HFD-induced obese mice were better able to defend their body temperature during environmental cold $\left(22^{\circ} \mathrm{C}\right)$ and acute cold stress $\left(4^{\circ} \mathrm{C}\right)$ compared to the control (Figure 4F-H, Figure 4-figure supplement 3H). Meanwhile, the Ang-(1-7) treated db/db mice had increased multi-locular structures but smaller lipid droplets, and increased UCP1 expression comparing to the control group (Figure 4I). Accordingly, the Ang-(1-7) treated $\mathrm{db} / \mathrm{db}$ and the HFD-induced obese mice showed more ${ }^{18} \mathrm{~F}$-FDG uptake in the BAT than the control mice recorded by PET-CT (Figure 4J, K).

The protein levels of UCP1 and PGC-1a were significantly induced in the BAT from the Ang-(1-7) treated db/db and the HFD-induced obese mice (Figure 4L, $M$ ). The mRNA levels, including UCP1, PGC-1a, Cidea, ATPsyn, COX4, COX8b, COX7a, MCAD and AP2, were increased in the BAT from the Ang-(1-7) treated $\mathrm{db} / \mathrm{db}$ mice (Figure 4-figure supplement 3).

To sum up, our results suggested that the enhanced thermogenesis effect in the Ad-ACE2 and Ang-(1-7) treated mice is caused by the increment of Ang-(1-7) levels, which demonstrates that Ang-(1-7) is crucial to the maintenance of thermogenesis.

\section{Ablation of Mas impairs thermogenesis in brown adipose tissue}

Since the Ang-(1-7), produced by ACE2, realized the function through the Mas receptor, these results above prompted us to hypothesize that the Mas receptor 


\section{9 / 37}

1 determines the effect of Ang-(1-7) in brown adipose tissue. Firstly, the

2 HFD-induced Mas-/mice (low Ang-(1-7) action model) were used to assess the

3 therapeutic effects (interventional effects) of Mas on energy metabolism. Although serum Ang-(1-7) levels were increased, the $\mathrm{Mas}^{-/}$mice had an impaired glucose tolerance, abnormal lipid profiles (Figure 4-figure supplement 4A-D), and significantly increased body weight compared to the WT mice (Figure 4-figure supplement 4E). Meanwhile, the $\mathrm{Mas}^{-/}$mice exhibited decreased oxygen consumption $\left(\mathrm{VO}_{2}\right)$ (Figure 4-figure supplement 4F) without any changes in food and/or water intake as well as physical activity (Figure 4-figure supplement 4G-I). Moreover, the $\mathrm{Mas}^{-/}$mice had lower thermogenesis than the WT mice in either $22{ }^{\circ} \mathrm{C}$ or $4{ }^{\circ} \mathrm{C}$ (Figure 4-figure supplement 3J-L). PET-CT analysis illustrated that the Mas $^{-/}$mice has less ${ }^{18} \mathrm{~F}-\mathrm{FDG}$ uptake in BAT than the WT mice (Figure 4-figure supplement 4M). Consistently, the $\mathrm{Mas}^{-/}$mice displayed larger lipid droplets and reduced multilocular structures, and had reduced UCP1 expression compared with the WT mice (Figure 4-figure supplement $4 \mathrm{M}$ ). Nevertheless, deletion of Mas resulted in a striking repression of BAT thermogenic protein (UCP-1, UQCRC2 and SDHB) (Figure 4-figure supplement 40) and genes (e.g. UCP1, PRMD16, PGC-1a, PGC-1ß, ATPsyn,

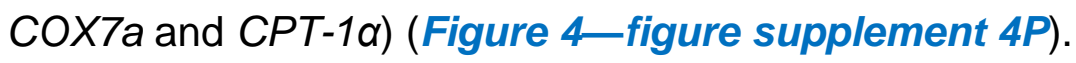

To investigate the role of the Mas receptor in BAT, we generated BAT specific Mas knockout mice ( $\mathrm{Mas}^{-/}$BAT transplanted mice). According to the previous studies (X. Liu et al., 2013; Yuan et al., 2016), firstly, BAT of the C57B/L6 recipient mice was removed from the interscapular region. Then, the BAT, which dissected from strain-, sex- and age-matched $\mathrm{Mas}^{-/}$donor mice, was subcutaneously transplanted into the dorsal interscapular region of the C57B/L6 recipient mice (WT+Mas ${ }^{-1-B A T)}$. As controls, C57B/L6 recipient mice transplanted with C57B/L6 
$10 / 37$

1 BAT (WT+WT-BAT) and C57B/L6 eWAT (WT+WT-eWAT) were used as positive

2 control and negative control, respectively. After the transplantation, the recipient

3 mice were fed by HFD for 10 weeks.

Interestingly, compared with the WT+WT-BAT control mice, the WT+Mas ${ }^{-}$-BAT mice showed greatly impaired HFD-induced insulin resistance. There is no significant difference between the WT+Mas $\%$-BAT mice and the WT+WT-eWAT mice in intraperitoneal glucose tolerance test (GTT) and the insulin tolerance test (ITT) (Figure 4-figure supplement 4Q, R). Notably, Mas`-BAT transplantation also strikingly induced HFD-induced weight gain in the WT+Mas ${ }^{--B A T}$ mice compared with the WT+WT-BAT control mice (Figure 4-figure supplement $4 S)$.

More importantly, compared to the WT+WT-BAT control mice, the $\mathrm{WT}+\mathrm{Mas}^{-/-\mathrm{BAT}}$ mice had decreased oxygen consumption $\left(\mathrm{VO}_{2}\right)$, carbon dioxide release $\left(\mathrm{VCO}_{2}\right)$ and energy expenditure (EE) (Figure 4-figure supplement $4 T-V$, along with normal food and/or water intake as well as physical activity (Figure 4-figure supplement $4 W-\eta$ ). Taken together, these results demonstrate that the Mas receptor can directly induce thermogenic program in brown adipose tissues.

\section{ACE2 pathway induces white fat browning and thermogenesis}

Next, we investigated the impact of ACE2/Ang-(1-7) on the process of browning of WAT, a prominent feature in scWAT. Histological examination of scWAT from the Ad-ACE2-treated $\mathrm{db} / \mathrm{db}$ obese mice showed a profound morphological transformation towards a BAT-like phenotype (smaller adipocytes with multiple lipid droplets) compared with the control (Figure 5A). Meanwhile, markers of brown adipocytes, such as UCP1, were significantly increased in scWAT of the Ad-ACE2-treated db/db mice. A much greater induction of transcription factors, 
$11 / 37$

1 including PRMD16, $P G C-1 \alpha$ and $P G C-1 \beta$, occurred in the scWAT of the

2 Ad-ACE2-treated group (Figure 5B). As expected, the Ang-(1-7) treated db/db

3 mice had a similar browning effect as the Ad-ACE2-treated db/db mice in scWAT.

4 Morphological brown-like adipocyte and thermogenic gene expression levels

5 slight increasement were observed in the scWAT after Ang-(1-7) treatment

6 (Figure 5C, D).

7 These alterations were restricted to scWAT, but not to eWAT. As shown in Fig.

$85 e$, the morphology and size of eWAT in the Ad-ACE2-treated $\mathrm{db} / \mathrm{db}$ mice are

9 same compared to the control (Figure 5E). Meanwhile, no significant increase in

10 the thermogenic gene expression levels in the eWAT of the Ad-ACE2-treated

$11 \mathrm{db} / \mathrm{db}$ mice was found (Figure $5 F$ ).

\section{ACE2 pathway enhances thermogenesis via Akt and PKA signaling}

We further investigated the molecular mechanisms through which ACE2 pathway regulates BAT. Firstly, we performed RNA-seq analysis on BAT isolated from the WT and the ACE2 KO mice. Notable differences between the two are displayed as 3D-PCA analysis and heat map (Figure 6-figure supplement 5A, B). Consistent with the RT-PCR results of BAT in the ACE2 KO mice, genetic deficiency of ACE2 significantly altered expression of genes involved in fatty acid biosynthesis, lipid catabolism, lipid biosynthesis, fatty acid beta-oxidation and cholesterol biosynthesis in the BAT of the ACE2 KO mice (Figure 6-figure supplement 5 C).

Interestingly, we found that the expression level of Akt associated genes were significantly decreased in the ACE2 KO mice compared with the WT mice (Figure 6-figure supplement 5D). The phosphorylation levels of Akt at residues Thr308 was significantly inhibited in BAT of the ACE2 KO mice (Figure 6A). Furthermore, 
12 / 37

1 the phosphorylation levels of Akt were dramatically increased in BAT of the

2 Ad-ACE2 treated mice (Figure 6B).

These results prompted us to consider whether ACE2 pathway regulates the function of BAT via Akt signaling. Thus, we treated primary brown adipocytes which isolated from mice with Ang-(1-7). We found phosphorylation of Akt was activated by Ang-(1-7), accompanied by UCP1 up-regulation and forkhead box-containing protein $\mathrm{O}$ subfamily-1 (FoxO1) phosphorylation (Figure 6C). MK2206, an Akt inhibitor (Chorner \& Moorehead, 2018; Matsuzaki, Pouly, \& Canis, 2018), suppressed Ang-(1-7) induced UCP1 up-regulation and FoxO1 phosphorylation (Figure 6C). Compared to Ang-(1-7) treated primary brown adipocytes cells, MK2206 down-regulated the mRNA levels of UCP1, PGC-1a, Cidea, ATPsyn, AP2 and CPT-1a genes (Figure 6D). More importantly, Ang-(1-7) treated primary brown adipocytes exhibited higher OCR, and MK2206 inhibited OCR in the Ang-(1-7) treated primary brown adipocytes (Figure 6E). Accordingly, the ACE2-overexpressing primary brown adipocytes cells showed a similar result. MK2206 suppressed ACE2 induced up-regulation of protein (UCP1 and phosphorylated FoxO1) (Figure 6-figure supplement 6A) and mRNA (UCP1 and CPT-1a) (Figure 6-figure supplement 6B), as well as action on OCR (Figure 6-figure supplement 6C). These results suggest that the Akt signaling are required for the thermogenic activity of ACE2 pathway.

After the determination that ACE2 pathway regulates adaptive thermogenesis through the Akt signaling, we paid attention on whether this program could still be provoked by protein kinase A (PKA) signaling, a pathway known to be involved in the canonical thermogenic activation of fat cells. Interestingly, we found that the phosphorylation level of PKA was significantly inhibited in BAT of the ACE2 KO mice (Figure 6F). Similar results appeared in the Mas KO mice (Figure 6-figure 
13 / 37

1 supplement 6D). However, the phosphorylation level of PKA was increased in

2 BAT of the Ad-ACE2-treated db/db mice (Figure 6G).

To further elucidate the study on the mechanism of Ang-(1-7)-induced PKA signaling, we treated Ang-(1-7)-treated primary brown adipocytes with PKA and adenylylcyclase inhibitors, simultaneously. Firstly, the Ang-(1-7)-induced PKA signaling was validated in primary brown adipocytes (Figure 6-figure supplement 6E). However, H89, a PKA inhibitor, significantly blunted the Ang-(1-7)-induced mRNA levels (UCP1, Cidea and AP2) (Figure 6H) and protein levels (UCP1 and PGC-1a) (Figure 6I). Similar effects were observed by using SQ-22536, an adenylylcyclase inhibitor on the formation of intracellular cAMP (Figure $6 \mathrm{H}, \mathrm{I})$. As expected, the Ang-(1-7) treated primary brown adipocytes exhibited higher OCR, and H89 inhibited OCR in the Ang-(1-7) treated primary brown adipocytes (Figure 6J). Our results thus strongly suggest that the PKA signaling is important for the thermogenic activity of ACE2 pathway.

\section{Discussion}

RAS is classically known to regulate blood pressure and maintain water and electrolyte balance. It also plays as a crucial role in metabolic disorders, such as obesity and insulin resistance (Das, 2016). Comprehensive understanding of the complexly biological function of the RAS remains a major biomedical challenge. In the ACE2 pathway, ACE2 is the primary function element, which determines the content of Ang-(1-7), a direct acting peptide, while MAS receptor determines the action form of Ang-(1-7). In order to clarify the role and mechanism of the ACE2 pathway in regulation of thermogenesis, it is necessary to systematically analyze the function of these three elements. 
14 / 37

In this study, we used six mice models, ACE2 KO mice, Ad-ACE2 db/db mice, Ang-(1-7) treated db/db and HFD mice, Mas KO mice and BAT-specific Mas knockout mice (Mas ${ }^{-1}$ BAT transplanted mice), respectively. Based on a series of functional assays in these six mouse models and primary brown adipocytes, we effectively confirmed that the ACE2 pathway regulates glucose and lipid homeostasis. Furthermore, ACE2 pathway maintains thermogenesis and systemic energy metabolism. Molecular analyses, including the use of several inhibitors of Akt and PKA, demonstrate that the effects of ACE2 pathway on brown adipocytes are mediated by both the Akt signaling and the PKA signaling, resulting in the activation of PGC-1 $\alpha$, followed by activation of UCP1 (Figure $6 K$ ). These findings significantly expand our understanding of the biological function of RAS. Furthermore, our results propose a new concept that the ACE2 pathway can improve obesity and the associated metabolic disorders.

BAT, which utilizes glucose and fatty acids for thermogenesis, contains large number of mitochondria and promotes thermogenesis by mitochondrial respiration through UCP1. BAT-specific UCP1, localized in the inner mitochondrial membrane, plays a fundamental role in thermogenesis. In response to stimulation, activation of PGC-1a up-regulates the expression of BAT-specific UCP1, which dissipates the proton motive force across the inner mitochondrial membranes, and consequentially producing ATP (Sambeat et al., 2016; Schreiber et al., 2017). On the other hand, PGC-1 $\alpha$ induces the acquisition of BAT features, including the expression of mitochondria and fatty acid-oxidation and thermogenic genes (Puigserver et al., 1998; Tiraby et al., 2003). We found that the mRNA levels of UCP1, $P G C-1 \alpha$, mitochondrial program and fatty acid oxidation related genes

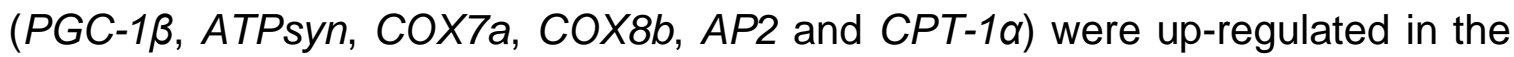
ACE2 overexpression and the Ang-(1-7) treated $\mathrm{db} / \mathrm{db}$ mice, whereas 
15 / 37

1 down-regulated in the ACE2 KO or the Mas KO mice. These results supported

2 that $P G C-1 \alpha$ and UCP1 might be critical for the effects of ACE2 pathway on

3 thermogenesis.

We also investigated the underlying mechanisms of ACE2 pathway on the regulation of BAT via the Akt signaling and the PKA signaling (Figure $6 K$ ).

First, we verified the Akt signaling in the downstream of ACE2 pathway. Akt has a critical function in cell survival and energy balance. Multiple pieces of evidence show that activation of PI3K is followed by the activation of Akt, which in turn triggers a complex cascade of events that include the inhibition of FoxO1 transcription factors and thus the activation of UCP1 and its transcriptional regulator PGC1a (Nakae et al., 2008; Ortega-Molina et al., 2012). In human and 3T3-L1 preadipocytes, Ang-(1-7)-Mas signaling promotes adipogenesis via activation of PI3K-Akt signaling (Than, Leow, \& Chen, 2013). AT2R activation induces white adipocyte browning by increasing PPARy expression, at least in part, via PI3K-Akt signaling pathways (Than et al., 2017). We previously reported that ACE2 and Ang-(1-7) can activate Akt signaling to ameliorate hepatic steatosis (Cao et al., 2016; Cao et al., 2014). In the present study, the ACE2 KO and the Mas KO mice displayed a strong decrease in Akt S308 phosphorylation in BAT. The ACE2 over-expression or the Ang-(1-7) treatment activated Akt S308 phosphorylation in BAT. Furthermore, the effect of ACE2-Ang-(1-7) on primary brown adipocytes can be attenuated by Akt inhibitor. These results suggest that the Akt signaling might also play a role in ACE2 pathway related regulation of BAT function.

Second, we verified the PKA signaling in the downstream of ACE2 pathway. The Mas receptor was shown to constitutively couple to Gas, including Gai, Gaq and Ga12/13proteins (Dias-Peixoto et al., 2008; Gomes, Santos, \& Guatimosim, 
$16 / 37$

1 2012; Tirupula, Desnoyer, Speth, \& Karnik, 2014). In the kidney, Ang-(1-7)

2 treatment increased cAMP levels and activated PKA through Gas activation by

3 the Mas receptor (G. C. Liu, Oudit, Fang, Zhou, \& Scholey, 2012; Magaldi, Cesar,

4 de Araujo, Simoes e Silva, \& Santos, 2003). Ang-(1-7) regulates insulin secretion

5 through a Mas-dependent cAMP signaling pathway (Sahr et al., 2016). It is well

6 known that norepinephrine released from the sympathetic nerves is a powerful

7 stimulator of BAT. Norepinephrine activates BAT thermogenic program via PKA

8 signaling, followed by the UCP1-mediated proton uncoupling (Su et al., 2017). In

9 this study, the PKA signaling in BAT was changed significantly by ACE2 pathway

10 in mice model. In addition, the effect of ACE2 pathway on primary brown

11 adipocytes can be depressed by cAMP and PKA inhibitor.

Previously, we demonstrate that the ACE2 pathway is involved in the regulation of glucose and lipid homeostasis with limited understanding of the underlying mechanisms (Cao et al., 2014; C. Liu et al., 2012; Niu et al., 2008; Shi et al., 2018;

F. Zhang et al., 2016). Here, for the first time, we provide evidence that the alteration in glucose and lipid homeostasis is associated with the change in maintaining brown adipocyte function for the facilitation of energy expenditure. In summary, the ACE2 pathway regulates BAT function and systemic energy metabolisms which is a potential treatment target for metabolic disorders including metabolic syndrome, diabetes, dyslipidemia and fatty liver.

\section{Materials and methods}

Mice

Obese C57BLKS/J-Leprdb/Leprdb (db/db) male mice, wild-type mice and Mas KO mice were purchased from Nanjing Biological Medicine Research Institute, Nanjing University, China. Male C57BL/6J mice were purchased from Vital River 
17 / 37

1 Laboratory Animal Technology (Beijing, China). ACE2 KO mice have been

2 previously described (Niu et al., 2008).

The obese diabetic $\mathrm{db} / \mathrm{db}$ mice at 7-8 weeks of age were used. Adenovirus $\left(5 \times 10^{8}\right.$ particle forming units $(\mathrm{pfu})$ in a total volume of $100 \mu \mathrm{L}$ of $0.9 \% \mathrm{wt} / \mathrm{vol}$ saline) was introduced into the $\mathrm{db} / \mathrm{db}$ mice by tail vein injection. The ad-ACE2 $\mathrm{db} / \mathrm{db}$ mice were used at the 6th day post-virus injection. The $\mathrm{db} / \mathrm{db}$ mice were treated with Ang-(1-7) by subcutaneous infusion of Ang-(1-7) (Sigma-Aldrich, St. Louis, MO) (100 ng/kg/min) or saline using osmotic mini-pumps (Alzet-Durect, Cupertino, CA, USA Model \#1004) for 4 weeks.

6-week-old male C57BL/6J mice were used to develop obesity by high-fat (HFD) diet (60 kcal\% fat) (Research Diets, New Brunswick, NJ, USA) for 8 weeks, and the mice treated with Ang-(1-7) by osmotic mini-pumps at the 5th weeks post-HFD. 8- to 10-weeks old male ACE2 KO mice and WT controls, Mas KO and WT controls were fed HFD diet for 8 weeks before experimental analysis. The mice were housed in a room at controlled temperature $\left(23^{\circ} \mathrm{C} \pm 1^{\circ} \mathrm{C}\right)$ with a 12 -hour light-dark cycle. All animals were handled in accordance with the protocol approved by the Ethics Committee of Animal Research at Beijing Tongren Hospital, Capital Medical University, Beijing, China.

\section{BAT transplantation}

According to the methods described previously (X. Liu et al., 2013; Yuan et al., 2016), BAT was removed from the interscapular region of 8-week old Mas KO mice or $\mathrm{C} 57 \mathrm{BL} / 6$ mice donor mouse and implanted into the interscapular region of recipient mice. BAT of $\mathrm{C}$ 57B/L6 recipient mice was removed from the interscapular region. After cervical dislocation of donor mice, the BAT or eWAT (also from the epididymal fat pad of 8-week-old C57BL/6 mice) was removed and peripheral white fat was excluded, and then the remaining BAT $(0.2 \mathrm{~g})$ or eWAT 
$18 / 37$

1 (0.2 g) was washed with sterile PBS and transplanted into the interscapular region

2 of recipients as quickly as possible. Recipient mice were anesthetized by ip

3 injection with $400 \mathrm{mg} / \mathrm{kg}$ body weight avertin, and then BAT or eWAT was

4 transplanted underneath the skin. The recipient mice were then fed an HFD,

5 which began immediately after the transplantation and continued for 10 weeks.

$6 \quad$ Adipocyte oxygen consumption rate (OCR) measurement

$7 \quad$ Primary brown adipose cells were isolated and cultured for 3 days before plated in

8 XF cell culture microplates (Seahorse Bioscience). Cells (10,000 cells) were

9 seeded in each well and each sample has 8 replicates. After 6 days of

10 differentiation, cultured adipocytes were washed twice and pre-incubated in XF

11 medium for $1-2 \mathrm{~h}$ at room temperature. The oxygen consumption rate was measured by the XF extracellular flux analyzer (Seahorse Biosciences). The chemicals (final concentration, $0.2 \mathrm{mM}$ Palmitate: $34 \mu \mathrm{M}$ BSA, $2 \mu \mathrm{M}$ Rotenone) were preloaded into cartridges and injected into XF wells in succession. OCR was calculated as a function of time (picomoles per minute).

\section{Glucose tolerance test (GTT)}

17 Mice were fasted for 16 hours (17:00-9:00) with free access to drinking water. Glucose $(1.0 \mathrm{~g} / \mathrm{kg}$ for the $\mathrm{db} / \mathrm{db}$ mice and $2.0 \mathrm{~g} / \mathrm{kg}$ for the HFD mice) was administered intraperitoneally (i.p.), and blood glucose levels were measured immediately $0,15,30,60$, and 120 min after glucose injection by using an Accu-Chek glucose monitor (Roche Diagnostics Corp).

\section{Glucose tolerance test (ITT)}

ITT was performed by injecting intraperitoneally $0.75 \mathrm{IU} / \mathrm{kg}$ of insulin at mice fasted for 1 hour and measured blood glucose levels at $0,15,30,60,90$, and 120 minutes post injection by using an Accu-Chek glucose monitor (Roche Diagnostics Corp). 
19 / 37

1 RNA extraction and quantitative Real-time RT-PCR

2 Total RNA was isolated using TRIzol reagent (Invitrogen, Carlsbad, CA, USA)

3 according to the manufacturer's instructions. A total of 500ng of RNA was used as

4 the template for the first-strand cDNA synthesis using ReverTra Ace qPCR RT Kit

5 (TOYOBO, Osaka, Japan) in accordance with the manufacturer's protocol. The

6 transcripts were quantified using Light Cycler 480Real-Time PCR system (Roche,

7 Basel, Switzerland). Primers were designed using Primer Quest (Integrated DNA

8 Technologies, Inc).

9 Positron emission tomography-computed tomography (PET-CT)

10 Siemens Inveon Dedicated PET (dPET) System and Inveon Multimodality (MM)

11 System (CT/SPECT) (Siemens Preclinical Solutions) was used to detect PET-CT imaging at Chinese Academy of Medical Sciences. According to the previously studies (X. Liu et al., 2013; Yuan et al., 2016), mice were allowed to fast overnight and were lightly anesthetized with isoflurane, and then followed by a tail vein injection of $18 \mathrm{~F}-\mathrm{FDG}(500 \mathrm{mCi})$. Sixty mins after the injection of the radiotracer, the mice were subjected to PET/CT analysis. A 10-min CT X-ray for attenuation correction was scanned before PET-CT scan. Static PET-CT scans were acquired for 10 minutes, and images were reconstructed by an OSEM3D algorithm followed by Maximization/Maximum a Posteriori (MAP) or Fast MAP provided by Inveon Acquisition Workplace (IAW) software. The 3D regions of interest (ROIs) were drawn over the guided CT images, and the tracer uptake was measured using Inveon Research Workplace (IRW) (Siemens) software. Individual quantification of the 18F-FDG uptake in each of the ROI was calculated. The data for the accumulation of 18F-FDG on micro PET images were expressed as the standard uptake values (SUVs), which were determined by dividing the relevant 
$20 / 37$

1 ROI concentration by the ratio of the injected activity to the bodyweight. The data

2 are presented as the mean \pm SEM.

3 Western blot analysis

4 Tissues were dissolved in RIPA buffer $(150 \mathrm{mM}$ sodium chloride, $1.0 \%$ Triton

$5 \mathrm{X}-100,0.5 \%$ sodium deoxycholate, $0.1 \%$ SDS, $50 \mathrm{mM}$ Tris, protease and

6 phosphatase inhibitor mixture (Roche Diagnostics)). Protein concentrations were

7 determined using a BCA assay kit (Pierce Diagnostics). Protein was separated by

$810 \%(w t / v o l)$ SDS/PAGE, transferred to a PVDF membrane (Millipore), blocked in

$95 \%(\mathrm{wt} / \mathrm{vol})$ skim milk in TBST (0.02 M Trisbase, $0.14 \mathrm{M}$ Vehicle, 0.1\% Tween 20,

$10 \mathrm{pH} 7.4)$, and incubated with primary antibodies overnight at $4^{\circ} \mathrm{C}$ and then

11 incubated with secondary antibodies conjugated with HRP. The following primary antibodies were used: anti-UCP1 (ab10983, Abcam), anti-PGC1a (ab54481, Abcam), anti-OXPHOS (ab110413, Abcam), anti-Mas (AAR-013, alomone labs), anti-Akt (\#9272, cell signaling technology), anti-p-Akt308 (\#13038, cell signaling technology), anti-FOXO1 (\#2880, cell signaling technology), anti- p-FOXO1 (\#84192, cell signaling technology), anti-PKA (\#4782, cell signaling technology), anti-p-PKA (\#9621, cell signaling technology), anti-ACE2 (\#4335, cell signaling technology), and actin (\#4970, Cell Signaling Technology). Signals were detected with Super Signal West Pico Chemiluminescent Substrate (Pierce).

\section{Histology and immunofluorescence analysis}

Tissues fixed in $4 \%$ paraformaldehyde were sectioned after being paraffin embedded. Multiple sections were prepared and stained with hematoxylin and eosin for general morphological observations. Cells grown on poly-L-lysine (Sigma)-pretreated coverslips were fixed with $4 \%$ paraformaldehyde. Immunofluorescence staining was performed according to the standard protocol using the following antibodies and dilutions: UCP1 (1:100 dilution; Santa Cruz 
21 / 37

1 Biotechnologies), MitoTracker Red (1:1000 dilution; Invitrogen). Incubations were

2 performed overnight in a humidified chamber at $4^{\circ} \mathrm{C} .40$,

3 6-diamidino-2-phenylindole staining was used to mark the cell nuclei. The images

$4 \quad$ were acquired by microscope (DS-RI1; Nikon)

$5 \quad$ Metabolic rate and physical activity

6 Oxygen consumption and physical activity were determined at 12 wk of age with a

7 TSE LabMaster, as previously described (Chi \& Wang, 2011). Mice were

8 acclimated to the system for 20-24 hours, and then $\mathrm{VO}_{2}$ and $\mathrm{VCO}_{2}$ were

9 measured during the next 24 hours. Voluntary activity of each mouse was

10 measured with an optical beam technique (Opto-M3, Columbus Instruments,

11 Columbus, OH, USA) over 24 hours and expressed as 24 hours average activity.

12 Heat production and respiratory exchange ratio (RER) were then calculated (X.

13 Liu et al., 2013).

RNA-Seq analysis

Total RNA was extracted from ACE2 KO or WT primary brown adipocytes by

Trizol reagent (Invitrogen), respectively. Extracted RNA samples were sent to Novel Bioinformatics company (Shanghai, China) for RNA-seq. RNA with RIN>8.0 is right for cDNA library construction. The cDNA libraries were processed for the proton sequencing according to the commercially available protocols. Data were submitted to the GEO archive. Fisher's exact test was calculated to select the significant pathway, and the threshold of significance was defined by P-value and false discovery rate (FDR) (Dupuy et al., 2007).

\section{Infrared thermography and core temperature}

Mice were exposed to a cold chamber $\left(4^{\circ} \mathrm{C}\right)$ with one mouse per cage for up to 6 hours, with free access to food and water. An infrared digital thermographic camera was used to taken images (E60: Compact Infrared Thermal Imaging 
22 / 37

1 Camera; FLIR). The images were analyzed by FLIR Quick Report software (FLIR

2 ResearchIR Max 3.4; FLIR). A rectal probe connected to a digital thermometer

3 was used to measure core body temperature (Yellow Spring Instruments).

$4 \quad$ Statistical analysis

5 All of the data are presented as the mean \pm SD. The data were analyzed by

6 Student's t test or one-way ANOVA (with Bonferroni post-hoc tests to compare

7 replicate means) when appropriate. Statistical comparisons were performed using

8 Prism5 (GraphPad Software, San Diego, CA). P values less than 0.05 were

9 considered statistically significant. Representative results from at least three

10 independent experiments are shown unless otherwise stated.

12 Acknowledgements

13 This work was supported by grants from National Key R\&D Program of China 14 (2017YFC0909600) and National Natural Science Foundation of China $15(81561128015,81471014)$ to Jinkui Yang, National Natural Science Foundation of China (81670774, 82070850) and Beijing Natural Science Foundation (7162047) to Xi Cao.

\section{Author contributions}

Xi Cao, Tingting Shiand Chuanhai Zhang designed the experiments, performed the experiments and wrote the manuscript. Lini Song, Yichen Zhang, Jingyi Liu. and Fangyuan Yang performed the experiments, Wanzhu Jin and Amin Xu. designed the experiments. Charles $\mathrm{N}$ Rotimi contributed to the interpretation of results and wrote the manuscript. Jinkui Yang conceived the idea for the study, designed the experiments and wrote the manuscript. 
23 / 37

2 Additional information

3 Supplementary materials: Please refer to Supplementary Figure S1-S6.

4 Competing interests: The authors declare no competing interests.

5 Data Availability: Sources for materials used in this study are described in

6 Materials and Methods.

7

8 


\section{References}

Cao, X., Yang, F., Shi, T., Yuan, M., Xin, Z., Xie, R., Li, S., Li, H., \& Yang, J. K. (2016). Angiotensin-converting enzyme 2/angiotensin-(1-7)/Mas axis activates Akt signaling to ameliorate hepatic steatosis. Sci Rep, 6, 21592. doi: 10.1038/srep21592

Cao, X., Yang, F. Y., Xin, Z., Xie, R. R., \& Yang, J. K. (2014). The ACE2/Ang-(1-7)/Mas axis can inhibit hepatic insulin resistance. Mol Cell Endocrinol, 393(1-2), 30-38. doi: 10.1016/j.mce.2014.05.024

Chi, Q. S., \& Wang, D. H. (2011). Thermal physiology and energetics in male desert hamsters (Phodopus roborovskii) during cold acclimation. J Comp Physiol B, 181(1), 91-103. doi: 10.1007/s00360-010-0506-6

Chorner, P. M., \& Moorehead, R. A. (2018). A-674563, a putative AKT1 inhibitor that also suppresses CDK2 activity, inhibits human NSCLC cell growth more effectively than the pan-AKT inhibitor, MK-2206. 13(2), e0193344. doi: 10.1371/journal.pone.0193344

Clarke, N. E., \& Turner, A. J. (2012). Angiotensin-converting enzyme 2: the first decade. Int J Hypertens, 2012, 307315. doi: 10.1155/2012/307315

Das, U. N. (2016). Renin-angiotensin-aldosterone system in insulin resistance and metabolic syndrome. J Transl Int Med, 4(2), 66-72. doi: 10.1515/jtim-2016-0022

Dias-Peixoto, M. F., Santos, R. A., Gomes, E. R., Alves, M. N., Almeida, P. W., Greco, L., Rosa, M., Fauler, B., Bader, M., Alenina, N., \& Guatimosim, S. (2008). Molecular mechanisms involved in the angiotensin-(1-7)/Mas signaling pathway in cardiomyocytes. Hypertension, 52(3), 542-548. doi: 10.1161/HYPERTENSIONAHA.108.114280

Dong, M., Lin, J., Lim, W., Jin, W., \& Lee, H. J. (2017). Role of brown adipose tissue in metabolic syndrome, aging, and cancer cachexia. Front Med. doi: 10.1007/s11684-017-0555-2

Dupuy, D., Bertin, N., Hidalgo, C. A., Venkatesan, K., Tu, D., Lee, D., Rosenberg, J., Svrzikapa, N., Blanc, A., Carnec, A., Carvunis, A. R., Pulak, R., Shingles, J., Reece-Hoyes, J., Hunt-Newbury, R., Viveiros, R., Mohler, W. A., Tasan, M., Roth, F. P., Le Peuch, C., Hope, I. A., Johnsen, R., Moerman, D. G., Barabasi, A. L., Baillie, D., \& Vidal, M. (2007). Genome-scale analysis of in vivo spatiotemporal promoter activity in Caenorhabditis elegans. Nat Biotechnol, 25(6), 663-668. doi: $10.1038 /$ nbt1305

Gomes, E. R., Santos, R. A., \& Guatimosim, S. (2012). Angiotensin-(1-7)-mediated signaling in cardiomyocytes. Int J Hypertens, 2012, 493129. doi: 10.1155/2012/493129

Hashimoto, T., Perlot, T., Rehman, A., Trichereau, J., Ishiguro, H., Paolino, M., Sigl, V., Hanada, T., Hanada, R., Lipinski, S., Wild, B., Camargo, S. M., Singer, D., Richter, A., Kuba, K., Fukamizu, A., Schreiber, S., Clevers, H., Verrey, F., Rosenstiel, P., \& Penninger, J. M. (2012). ACE2 links amino acid malnutrition to microbial ecology and intestinal inflammation. Nature, 487(7408), 477-481. doi: 
Liu, C., Lv, X. H., Li, H. X., Cao, X., Zhang, F., Wang, L., Yu, M., \& Yang, J. K. (2012). Angiotensin-(1-7) suppresses oxidative stress and improves glucose uptake via Mas receptor in adipocytes. Acta Diabetol, 49(4), 291-299. doi: 10.1007/s00592-011-0348-z

Liu, G. C., Oudit, G. Y., Fang, F., Zhou, J., \& Scholey, J. W. (2012). Angiotensin-(1-7)-induced activation of ERK1/2 is CAMP/protein kinase A-dependent in glomerular mesangial cells. Am J Physiol Renal Physiol, 302(6), F784-790. doi: 10.1152/ajprenal.00455.2011

Liu, X., Zheng, Z., Zhu, X., Meng, M., Li, L., Shen, Y., Chi, Q., Wang, D., Zhang, Z., Li, C., Li, Y., Xue, Y., Speakman, J. R., \& Jin, W. (2013). Brown adipose tissue transplantation improves whole-body energy metabolism. Cell Res, 23(6), 851-854. doi: 10.1038/cr.2013.64

Magaldi, A. J., Cesar, K. R., de Araujo, M., Simoes e Silva, A. C., \& Santos, R. A. (2003). Angiotensin-(1-7) stimulates water transport in rat inner medullary collecting duct: evidence for involvement of vasopressin V2 receptors. Pflugers Arch, 447(2), 223-230. doi: 10.1007/s00424-003-1173-1

Matsuzaki, S., Pouly, J. L., \& Canis, M. (2018). In vitro and in vivo effects of MK2206 and chloroquine combination therapy on endometriosis: Autophagy may be required for regrowth of endometriosis. Br J Pharmacol. doi: 10.1111/bph.14170

Nakae, J., Cao, Y., Oki, M., Orba, Y., Sawa, H., Kiyonari, H., Iskandar, K., Suga, K., Lombes, M., \& Hayashi, Y. (2008). Forkhead transcription factor FoxO1 in adipose tissue regulates energy storage and expenditure. Diabetes, 57(3), 563-576. doi: 10.2337/db07-0698

Niu, M. J., Yang, J. K., Lin, S. S., Ji, X. J., \& Guo, L. M. (2008). Loss of angiotensin-converting enzyme 2 leads to impaired glucose homeostasis in mice. Endocrine, 34(1-3), 56-61. doi: $10.1007 / \mathrm{s} 12020-008-9110-x$

Ortega-Molina, A., Efeyan, A., Lopez-Guadamillas, E., Munoz-Martin, M., Gomez-Lopez, G., Canamero, M., Mulero, F., Pastor, J., Martinez, S., Romanos, E., Mar Gonzalez-Barroso, M., Rial, E., Valverde, A. M., Bischoff, J. R., \& Serrano, M. (2012). Pten positively regulates brown adipose function, energy expenditure, and longevity. Cell Metab, 15(3), 382-394. doi: 10.1016/j.cmet.2012.02.001

Puigserver, P., Wu, Z., Park, C. W., Graves, R., Wright, M., \& Spiegelman, B. M. (1998). A cold-inducible coactivator of nuclear receptors linked to adaptive thermogenesis. Cell, 92(6), 829-839.

Sahr, A., Wolke, C., Maczewsky, J., Krippeit-Drews, P., Tetzner, A., Drews, G., Venz, S., Gurtler, S., van den Brandt, J., Berg, S., Doring, P., Dombrowski, F., Walther, T., \& Lendeckel, U. (2016). The Angiotensin-(1-7)/Mas Axis Improves Pancreatic beta-Cell Function in Vitro and in Vivo. Endocrinology, 157(12), 4677-4690. doi: 10.1210/en.2016-1247

Sambeat, A., Gulyaeva, O., Dempersmier, J., Tharp, K. M., Stahl, A., Paul, S. M., \& Sul, H. S. (2016). LSD1 Interacts with Zfp516 to Promote UCP1 Transcription and Brown Fat Program. Cell Rep, 15(11), 2536-2549. doi: 10.1016/j.celrep.2016.05.019 
Santos, R. A., Simoes e Silva, A. C., Maric, C., Silva, D. M., Machado, R. P., de Buhr, I., Heringer-Walther, S., Pinheiro, S. V., Lopes, M. T., Bader, M., Mendes, E. P., Lemos, V. S., Campagnole-Santos, M. J., Schultheiss, H. P., Speth, R., \& Walther, T. (2003). Angiotensin-(1-7) is an endogenous ligand for the G protein-coupled receptor Mas. Proc Natl Acad Sci U S A, 100(14), 8258-8263. doi: $10.1073 /$ pnas. 1432869100

Schreiber, R., Diwoky, C., Schoiswohl, G., Feiler, U., Wongsiriroj, N., Abdellatif, M., Kolb, D., Hoeks, J., Kershaw, E. E., Sedej, S., Schrauwen, P., Haemmerle, G., \& Zechner, R. (2017). Cold-Induced Thermogenesis Depends on ATGL-Mediated Lipolysis in Cardiac Muscle, but Not Brown Adipose Tissue. Cell Metab, 26(5), 753-763.e757. doi: 10.1016/j.cmet.2017.09.004

Shi, T. T., Yang, F. Y., Liu, C., Cao, X., Lu, J., Zhang, X. L., Yuan, M. X., Chen, C., \& Yang, J. K. (2018). Angiotensin-converting enzyme 2 regulates mitochondrial function in pancreatic beta-cells. Biochem Biophys Res Commun, 495(1), 860-866. doi: 10.1016/j.bbrc.2017.11.055

Su, J., Wu, W., Huang, S., Xue, R., Wang, Y., Wan, Y., Zhang, L., Qin, L., Zhang, Q., Zhu, X., Zhang, Z., Ye, H., Wu, X., \& Li, Y. (2017). PKA-RIIB Deficiency Induces Brown Fatlike Adipocytes in Inguinal WAT and Promotes Energy Expenditure in Male FVB/NJ Mice. Endocrinology, 158(3), 578-591. doi: 10.1210/en.2016-1581

Than, A., Leow, M. K., \& Chen, P. (2013). Control of adipogenesis by the autocrine interplays between angiotensin 1-7/Mas receptor and angiotensin II/AT1 receptor signaling pathways. J Biol Chem, 288(22), 15520-15531. doi: 10.1074/jbc.M113.459792

Than, A., Xu, S., Li, R., Leow, M. S., Sun, L., \& Chen, P. (2017). Angiotensin type 2 receptor activation promotes browning of white adipose tissue and brown adipogenesis. Signal Transduct Target Ther, 2, 17022. doi: 10.1038/sigtrans.2017.22

Tiraby, C., Tavernier, G., Lefort, C., Larrouy, D., Bouillaud, F., Ricquier, D., \& Langin, D. (2003). Acquirement of brown fat cell features by human white adipocytes. J Biol Chem, 278(35), 33370-33376. doi: 10.1074/jbc.M305235200

Tirupula, K. C., Desnoyer, R., Speth, R. C., \& Karnik, S. S. (2014). Atypical signaling and functional desensitization response of MAS receptor to peptide ligands. PLoS One, 9(7), e103520. doi: 10.1371/journal.pone.0103520

Trayhurn, P., \& Wusteman, M. C. (1990). Lipogenesis in genetically diabetic (db/db) mice: developmental changes in brown adipose tissue, white adipose tissue and the liver. Biochim Biophys Acta, 1047(2), 168-174.

Yuan, X., Hu, T., Zhao, H., Huang, Y., Ye, R., Lin, J., Zhang, C., Zhang, H., Wei, G., Zhou, H., Dong, M., Zhao, J., Wang, H., Liu, Q., Lee, H. J., Jin, W., \& Chen, Z. J. (2016). Brown adipose tissue transplantation ameliorates polycystic ovary syndrome. Proc Natl Acad Sci U S A, 113(10), 2708-2713. doi: $10.1073 /$ pnas.1523236113

Zhang, F., Liu, C., Wang, L., Cao, X., Wang, Y. Y., \& Yang, J. K. (2016). Antioxidant effect of angiotensin (17) in 
bioRxiv preprint doi: https://doi.org/10.1101/2021.08.10.455823; this version posted August 10, 2021. The copyright holder for this preprint (which was not certified by peer review) is the author/funder, who has granted bioRxiv a license to display the preprint in perpetuity. It is made available under aCC-BY 4.0 International license.

27 / 37

1

2

3

4

5

6

the protection of pancreatic beta cell function. Mol Med Rep, 14(3), 1963-1969. doi: 10.3892/mmr.2016.5514

Zhang, Z., Zhang, H., Li, B., Meng, X., Wang, J., Zhang, Y., Yao, S., Ma, Q., Jin, L., Yang, J., Wang, W., \& Ning, G. (2014). Berberine activates thermogenesis in white and brown adipose tissue. Nat Commun, 5, 5493. doi: $10.1038 /$ ncomms6493

7 


\section{Figures}

Figure 1.

A

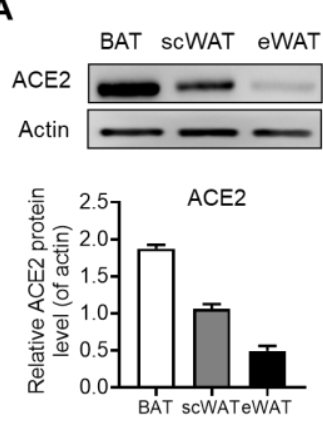

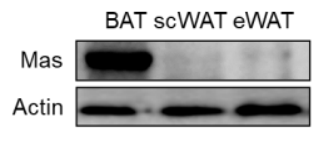

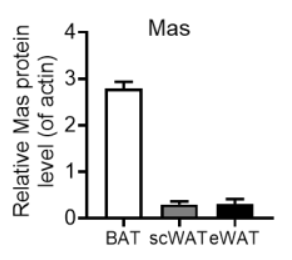

B

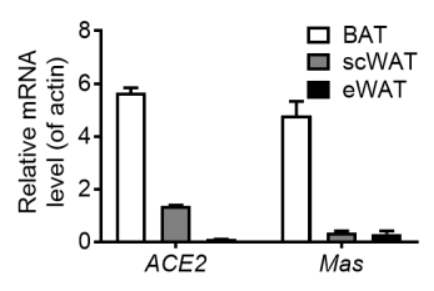

C

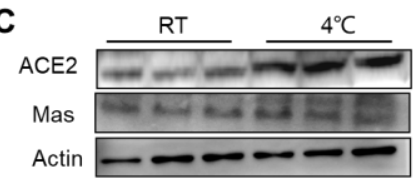

$\mathbf{F}$

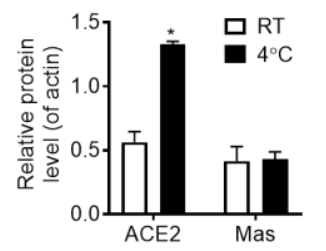

D

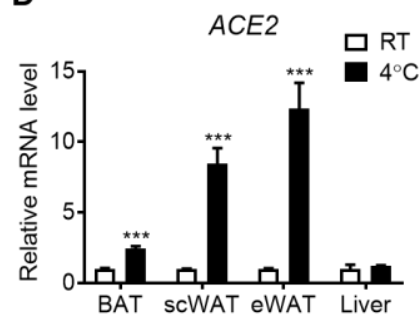

2

3

4 Figure 1. ACE2 pathway is activated by cold exposure.

5 Eight-week-old male C57BL/6J mice were housed at room temperature (RT) for 2 weeks 6 before cold exposure at $4^{\circ} \mathrm{C}$ for various time periods as indicated.

7 (A) Levels of ACE2 and Mas protein from interscapular brown adipose tissue (BAT), 8 subcutaneous and epididymal white adipose tissue (scWAT and eWAT) of C57BL/6 mice 9 at room temperature (RT), as determined by Western blotting. $n=3 /$ each group.

10 (B) Levels of ACE2 and Mas mRNA from BAT, scWAT and eWAT of C57BL/6 mice at RT, 11 as determined by $q$ PCR. $n=5 /$ each group.

12 (C) Levels of ACE2 and Mas protein from interscapular BAT of C57BL/6 mice at RT or 13 exposed to $4^{\circ} \mathrm{C}$ for 6 hours, as determined by Western blotting. $n=3$ /each group.

14 (D, E) Levels of ACE2 and Mas mRNA from BAT, scWAT, eWAT and liver of C57BL/6 mice exposed to $4^{\circ} \mathrm{C}$ for 24 hours, as determined by qPCR. $n=5 /$ each group. $(\mathbf{F}, \mathbf{G})$ Serum levels of ACE2 (f) and Ang-(1-7) $(\mathbf{g})$, as determined by ELISA. $n=6 /$ each group.

Data represent mean $\pm \mathrm{SEM} ;{ }^{*} p<0.05,{ }^{* *} p<0.01$ and ${ }^{* * *} p<0.001$ vs Control group by Student's $t$-test. 
A

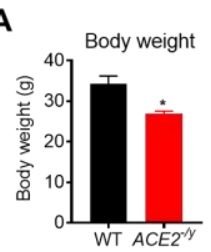

$\mathbf{F}$$$
\mathbf{F}
$$

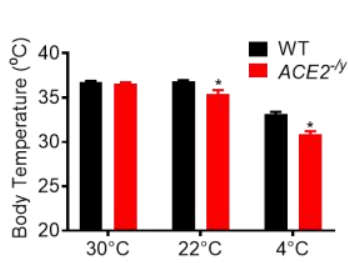

G

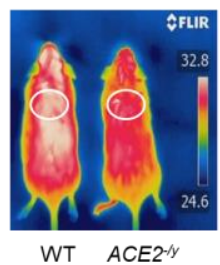

$\mathbf{J}$
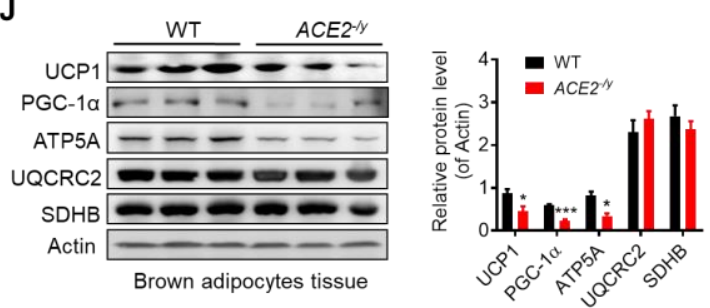

$\mathbf{L}$

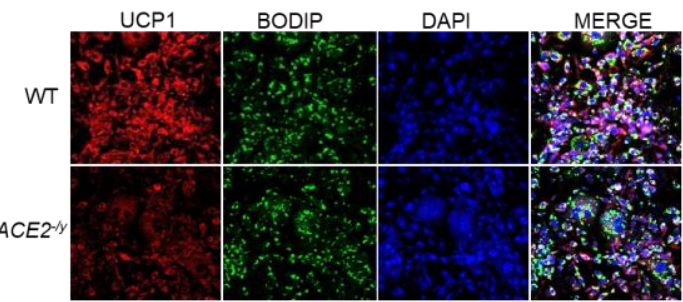

H
D
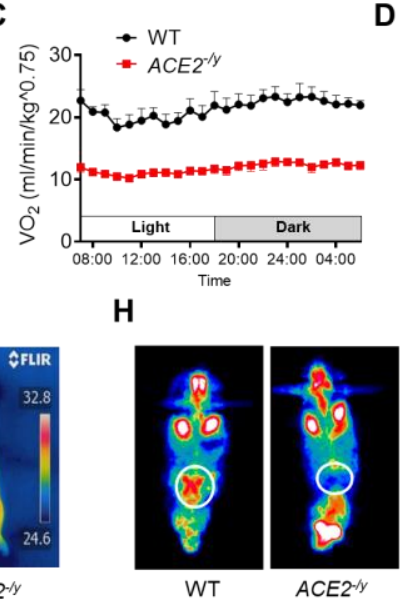

$\mathbf{K}$
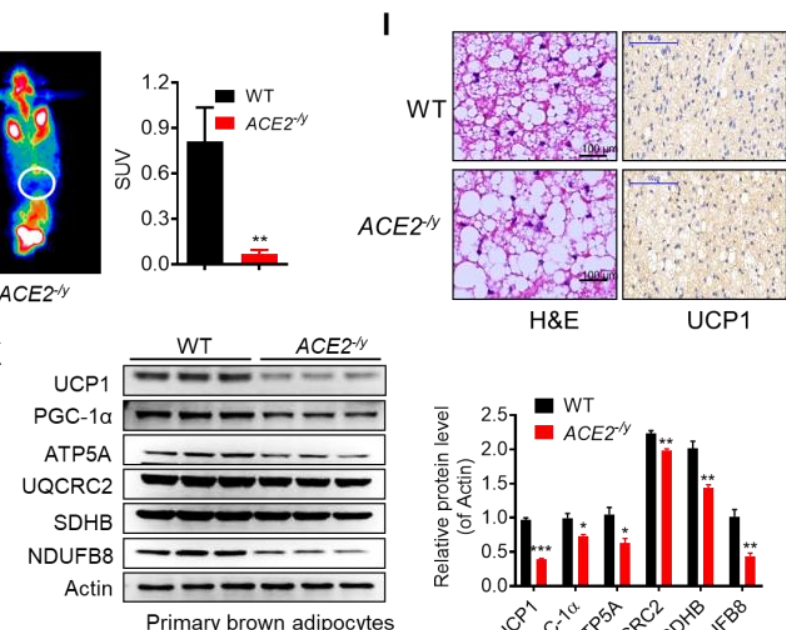

E

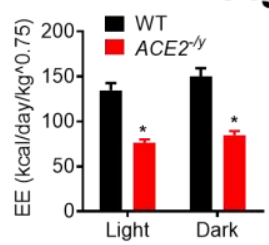

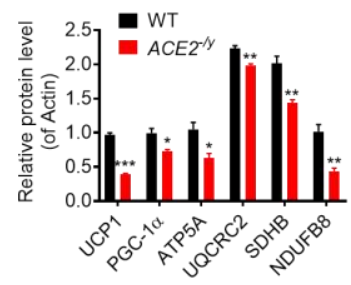

Figure 2.

M

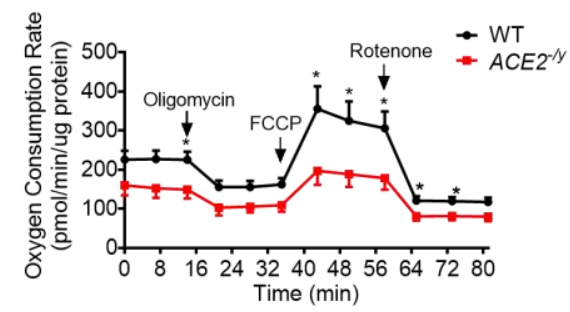

Figure 2. ACE2 deficiency impairs thermogenesis, brown adipose tissue (BAT) activity, and energy metabolism.

Eight-week-old male $A C E 2^{-1 y}$ mice and their wild type (WT) mice (controls) had a high-fat diet (HFD) for 8 weeks.

(A) Body weight of $A C E 2^{-1 y}$ and WT mice fed a HFD for 8 weeks.

(B) Serum levels of Ang-(1-7), as determined by ELISA.

(C-E) Energy expenditure was evaluated by measurement of oxygen consumption $\left(\mathrm{VO}_{2}\right)$

(C), carbon dioxide release $\left(\mathrm{VCO}_{2}\right)$ (D) and energy expenditure (EE) (E) over a $24 \mathrm{~h}$ period.

(F) Core body temperature at $30^{\circ} \mathrm{C}, 22^{\circ} \mathrm{C}$ and $4^{\circ} \mathrm{C}$ for 8 hours in $A C E 2^{-1 y}$ and WT mice.

(G) Infrared thermal images at $22^{\circ} \mathrm{C}$ in $A C E 2^{-y}$ and WT mice.

(H) Representative tomography-computed tomography (PET-CT) image and standard uptake values (SUVs).

(I) Representative haematoxylin and eosin (H\&E) staining and uncoupling protein-1 (UCP1) immunostaining from BAT sections of $A C E 2^{-y}$ and WT mice exposure at $4^{\circ} \mathrm{C}$.

(J) Representative western blots showing the changes of key proteins of energy expenditure and thermogenesis in BAT of $A C E 2^{-y}$ and WT mice exposure at $4^{\circ} \mathrm{C}(\mathrm{n}$ $=3$ /each group).

(K) Representative western blots showing the key protein changes in primary brown adipocytes from $A C E 2^{-1 y}$ and WT mice ( $\mathrm{n}=3$ /each group).

(L) Representative immunofluorescent images of in vitro differentiated primary brown adipocytes of $A C E 2^{-1 y}$ and WT mice, primary brown adipocytes show staining for UCP1 (red), boron-dipyrromethene (BODIPY) (green; neutral lipid dye), and DAPI (blue; nuclei). (M) Continuous measurement of oxygen consumption rate (OCR) in primary brown adipocytes from $A C E 2^{-y}$ mice and WT littermates. Oxygen consumption was performed 
30 / 37

1 under basal conditions, following the addition of oligomycin $(14 \mu \mathrm{M})$, the pharmacological 2 uncoupler FCCP $(10 \mu \mathrm{M})$ or the Complex III and I inhibitor antimycin A and rotenone $(4 \mu \mathrm{M}$ 3 each).

$4 \quad \mathrm{n}=5$-7/each group unless otherwise stated; Data represent mean $\pm \mathrm{SEM}$; ${ }^{*} p<0.05,{ }^{* *} p<$ $5 \quad 0.01$ vs WT group by Mann-Whitney U test.

6 The online version of this article includes the following figure supplement(s) for figure 2:

7 Figure supplement 1. ACE2 deficiency impairs adaptative thermogenesis by cold 8 stimulation. 
A

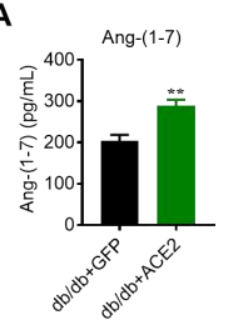

B

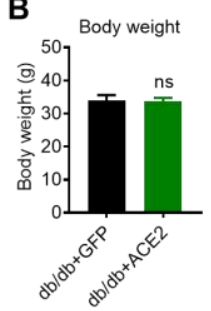

c

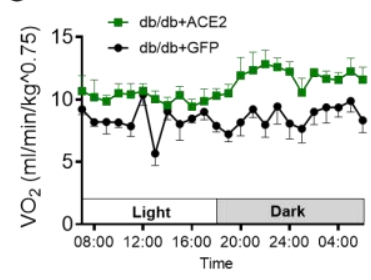

D

H

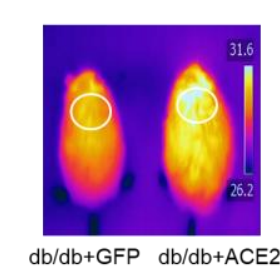

G

$d b / d b+G F P \quad d b / d b+A C E 2$

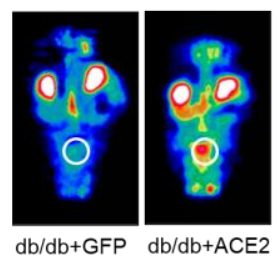

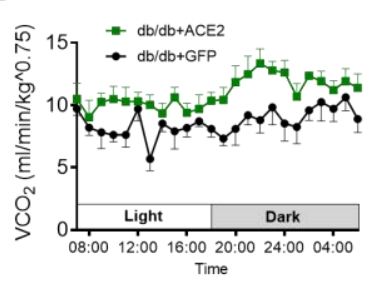

E

Figure 3.

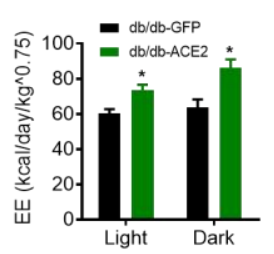

F

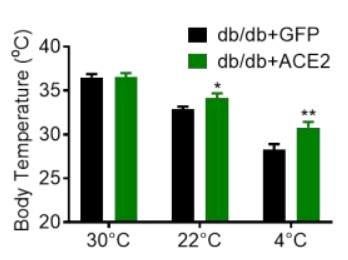

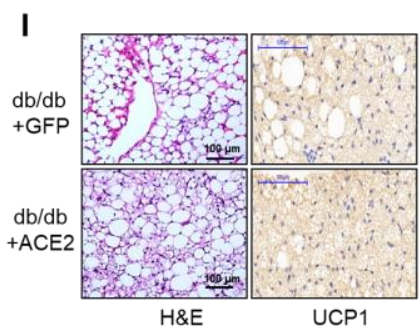

I

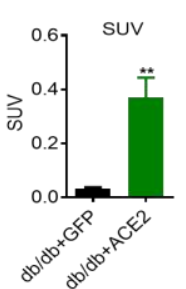

K

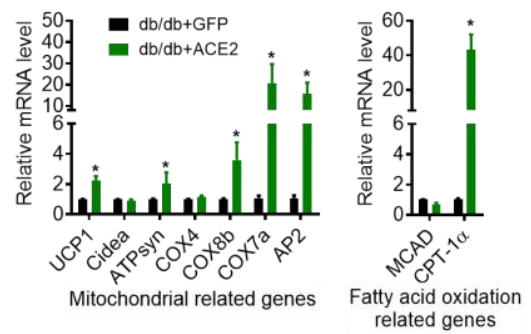

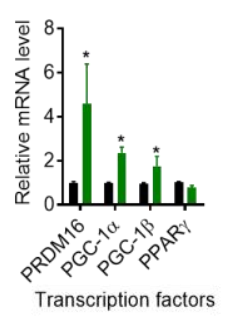

1

2

3

4

5

6

7

8

9

10

11

12

13

14

15

16

17

18

19

20

21

Figure 3. ACE2 enhances thermogenesis, brown adipose tissue (BAT) activity, and energy metabolism in $\mathrm{db} / \mathrm{db}$ obese mice.

ACE2 over-expression adenovirus (Ad-ACE2) and Ad-GFP (control) were introduced into the $\mathrm{db} / \mathrm{db}$ obese mice by tail vein injection. The ad-ACE2 and Ad-GFP treated $\mathrm{db} / \mathrm{db}$ mice were used at the 6th day post-virus injection.
(A) Serum levels of Ang-(1-7), as determined by ELISA
(B) Body weight of ad-ACE2 and Ad-GFP treated $\mathrm{db} / \mathrm{db}$ mice at the 6th day post-virus injection.

(C-E) Energy expenditure was evaluated by measurement of oxygen consumption $\left(\mathrm{VO}_{2}\right)$
(C), carbon dioxide release $\left(\mathrm{VCO}_{2}\right)$
(D) and energy expenditure (EE)
(E) over a $24 \mathrm{~h}$ period.

(F) Core body temperature at $30^{\circ} \mathrm{C}, 22^{\circ} \mathrm{C}$ and $4^{\circ} \mathrm{C}$ for 8 hours.

(G) Infrared thermal images at $22^{\circ} \mathrm{C}$ in $\mathrm{db} / \mathrm{db}+\mathrm{ACE} 2$ and $\mathrm{db} / \mathrm{db}+\mathrm{GFP}$ mice.

(H) Representative tomography-computed tomography (PET-CT) image and standard uptake values (SUVs).

(I) Representative H\&E staining and UCP1 immunostaining from BAT sections of $\mathrm{db} / \mathrm{db}+$ ACE2 $\mathrm{and} \mathrm{db} / \mathrm{db}+\mathrm{GFP}$ mice exposure at $4^{\circ} \mathrm{C}$.

(J) Representative western blots showing the changes of key proteins of energy expenditure and thermogenesis in BAT of $\mathrm{db} / \mathrm{db}+$ ACE2 and $\mathrm{db} / \mathrm{db}+\mathrm{GFP}$ mice exposure at $4^{\circ} \mathrm{C}$.

(K) Relative mRNA levels of mitochondrial related genes, fatty acid oxidation related genes and transcription factors in BAT of $\mathrm{db} / \mathrm{db}+\mathrm{ACE} 2$ and $\mathrm{db} / \mathrm{db}+\mathrm{GFP}$ mice exposure at $4^{\circ} \mathrm{C}$. 
32 / 37

$1 \mathrm{n}=5$-7/each group; Data represent mean $\pm \mathrm{SEM} ;{ }^{*} p<0.05,{ }^{* *} p<0.01$ vs Ad+GFP group

2 by Mann-Whitney $U$ test.

3 The online version of this article includes the following figure supplement(s) for figure 3:

4 Figure supplement 2. ACE2 enhance BAT activity and whole-body energy metabolism 5 in $\mathrm{db} / \mathrm{db}$ mice. 
A

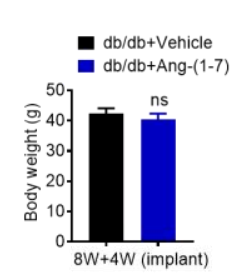

B

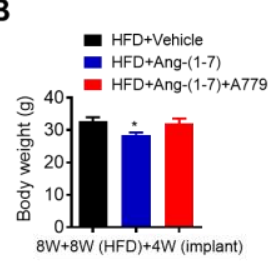

C

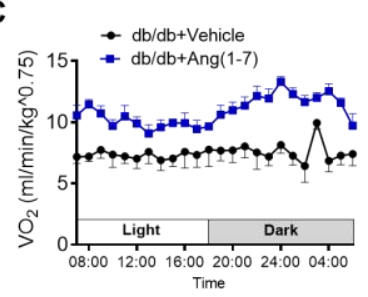

H

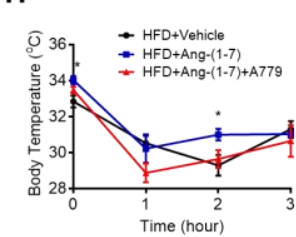

D

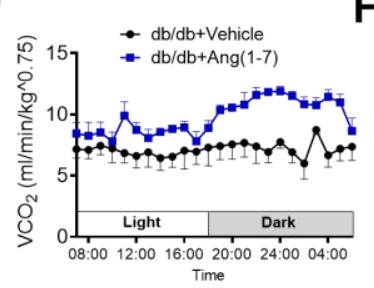

G
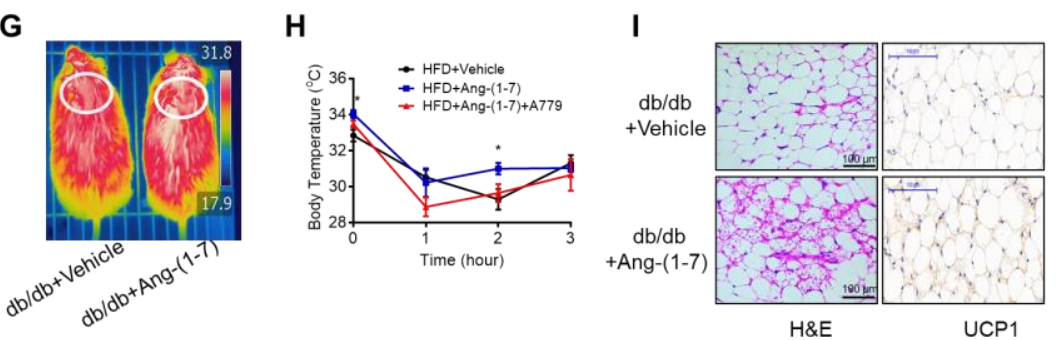

J
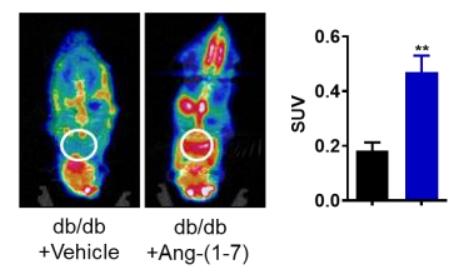

L
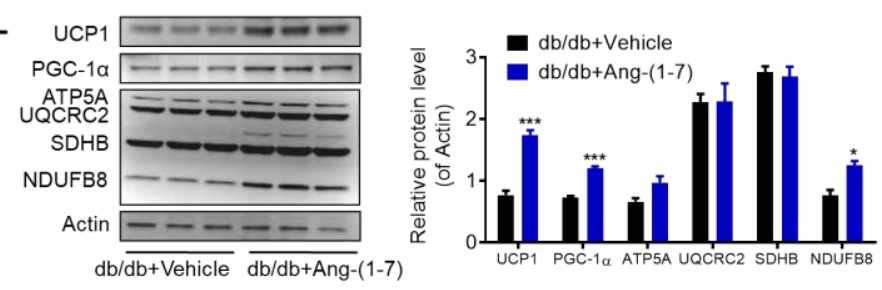

K
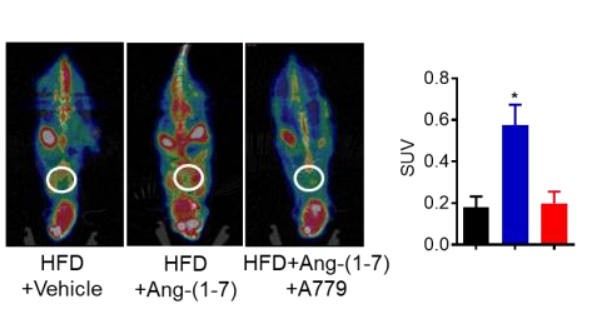

M

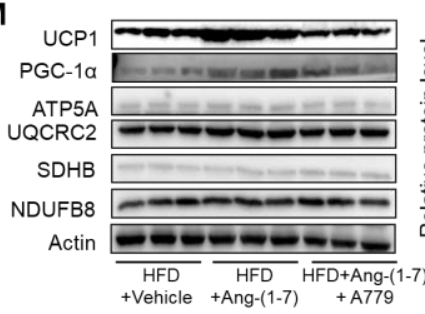

Figure 4.
3

4

5

6

7

8

9

10

11

12

13

14

15

16

17

Figure 4. Ang-(1-7) promotes thermogenesis, brown adipose tissue (BAT) activity, and energy metabolism in the $\mathrm{db} / \mathrm{db}$ and the HFD-induced obese mice.

Ang-(1-7) administration by subcutaneous implanted micro-osmotic pumps in the $\mathrm{db} / \mathrm{db}$ obese mice and the high-fat diet (HFD)-induced obese mice were used. The $\mathrm{db} / \mathrm{db}$ mice were treated with Ang-(1-7) by subcutaneous infusion of Ang-(1-7) or saline using osmotic mini-pumps for 4 weeks. 6-week-old male C57BL/6J mice were used to develop obesity by HFD diet for 8 weeks, and the mice treated with Ang-(1-7), A779 (an Ang-(1-7) antagonist) or saline by osmotic mini-pumps at the 4th weeks post-HFD.

(A) Body weight of $\mathrm{db} / \mathrm{db}+$ Ang-(1-7) and $\mathrm{db} / \mathrm{db}+$ Vehicle mice at the 4 th week post micro-osmotic pumps implantation.

(B) Body weight of HFD+Ang-(1-7), HFD+A779 and HFD+Vehicle mice at the 4th week post micro-osmotic pumps implantation.

(C-E) Energy expenditure was evaluated by measurement of oxygen consumption $\left(\mathrm{VO}_{2}\right)$ (c), of carbon dioxide release $\left(\mathrm{VCO}_{2}\right)(\mathbf{d})$ and of energy expenditure (EE) (e) over a $24 \mathrm{~h}$ period in $\mathrm{db} / \mathrm{db}+$ Ang-(1-7) and $\mathrm{db} / \mathrm{db}+$ Vehicle mice.

(F) Core body temperature at $30^{\circ} \mathrm{C}, 22^{\circ} \mathrm{C}$ and $4^{\circ} \mathrm{C}$ for 8 hours in $\mathrm{db} / \mathrm{db}+\mathrm{Ang}-(1-7)$ and $\mathrm{db} / \mathrm{db}$-Vehicle mice.

(G) Infrared thermal images at $22^{\circ} \mathrm{C}$ in $\mathrm{db} / \mathrm{db}+$ Ang-(1-7) and $\mathrm{db} / \mathrm{db}+$ Vehicle mice.

(H) Core body temperature at $4^{\circ} \mathrm{C}$ for the indicated lengths of time in HFD+Ang-(1-7), HFD+A779 and HFD+Vehicle mice.

(I) Representative H\&E staining and UCP1 immunostaining from BAT sections of $\mathrm{db} / \mathrm{db}+$ Ang-(1-7) and $\mathrm{db} / \mathrm{db}+$ Vehicle mice exposure at $4^{\circ} \mathrm{C}$.

(J) Representative Positron emission tomography-computed tomography (PET-CT) image and SUVs of $\mathrm{db} / \mathrm{db}+$ Ang-(1-7) and $\mathrm{db} / \mathrm{db}+$ Vehicle mice.

(K) Representative PET-CT image and SUVs of HFD+Ang-(1-7), HFD+A779 and HFD+Vehicle mice. 
1 (L) Representative western blots showing the changes of key proteins of energy expenditure and thermogenesis in BAT of $\mathrm{db} / \mathrm{db}+\mathrm{Ang}-(1-7)$ and $\mathrm{db} / \mathrm{db}+$ Vehicle mice exposure at $4^{\circ} \mathrm{C}$.

(M) Representative western blots showing the changes of key proteins of energy expenditure and thermogenesis in BAT of HFD+Ang-(1-7), HFD+A779 and HFD+Vehicle mice exposure at $4^{\circ} \mathrm{C}$. $\mathrm{n}=5-7 /$ each group; Data represent mean $\pm \mathrm{SEM} ;{ }^{*} p<0.05,{ }^{* \star} p<$ 0.01 vs Vehicle group by Student's $t$-test.

8 The online version of this article includes the following figure supplement(s) for figure 4:

9 Figure supplement 3. Ang-(1-7) promoted thermogenesis and energetic metabolism in 10 BAT of $\mathrm{db} / \mathrm{db}$ mice during cold challenge.

11 Figure supplement 4. Ablation of Mas impairs thermogenesis, BAT activity, and 12 energetic metabolism. 
A

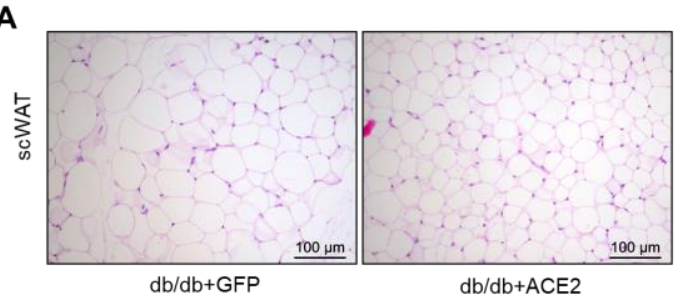

C

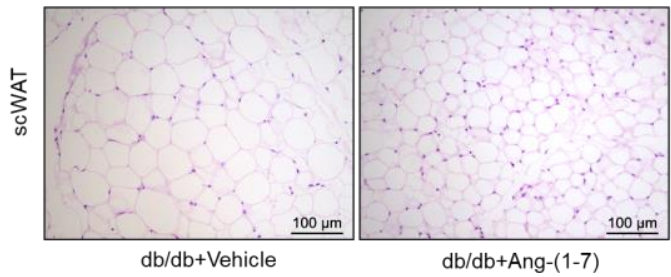

E

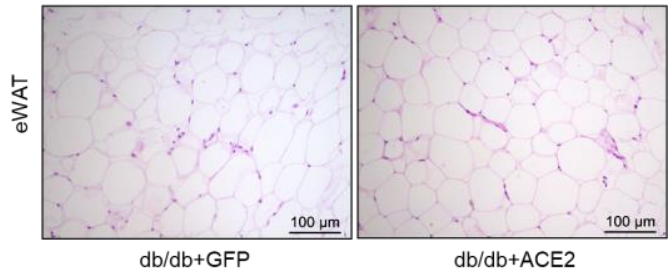

B

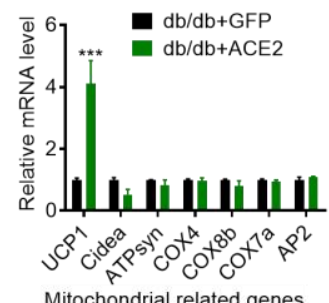

D

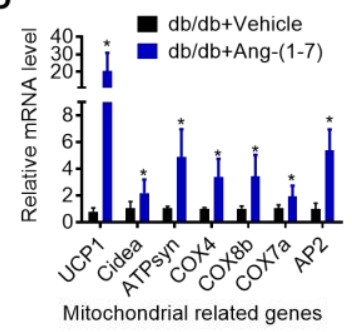

$\mathbf{F}$

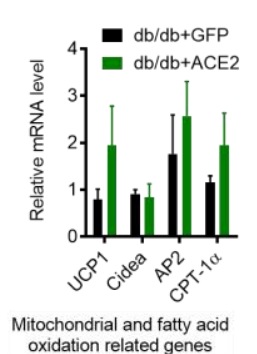

Figure 5.

1

2

3

4

5

6

7

8

9

10

11

Figure 5. ACE2 pathway induced white adipose tissue browning in the $\mathrm{db} / \mathrm{db}$ obese mice

(A) Representative H\&E staining from subcutaneous white adipose tissue (scWAT) sections of $\mathrm{db} / \mathrm{db}+\mathrm{ACE} 2 \mathrm{and} \mathrm{db} / \mathrm{db}+\mathrm{GFP}$ mice exposure at $4^{\circ} \mathrm{C}$.

(B) Relative mRNA levels of mitochondrial related genes, fatty acid oxidation related genes and transcription factors in scWAT of $\mathrm{db} / \mathrm{db}+\mathrm{ACE} 2 \mathrm{and} \mathrm{db} / \mathrm{db}+\mathrm{GFP}$ mice exposure at $4^{\circ} \mathrm{C}$.

(C) Representative H\&E staining from scWAT sections of $\mathrm{db} / \mathrm{db}+$ Ang-(1-7) and $\mathrm{db} / \mathrm{db}+$ Vehicle mice exposure at $4^{\circ} \mathrm{C}$.

(D) Relative mRNA levels of mitochondrial related genes, fatty acid oxidation related genes and transcription factors in scWAT of $\mathrm{db} / \mathrm{db}+A n g-(1-7)$ and $\mathrm{db} / \mathrm{db}+$ Vehicle mice exposure at $4^{\circ} \mathrm{C}$.

(E) Representative H\&E staining from epididymal white adipose tissue (eWAT) sections of $\mathrm{db} / \mathrm{db}+\mathrm{ACE} 2$ and $\mathrm{db} / \mathrm{db}+\mathrm{GFP}$ mice exposure at $4^{\circ} \mathrm{C}$.

(F) Relative mRNA levels of mitochondrial related genes, fatty acid oxidation related genes and transcription factors in eWAT of $\mathrm{db} / \mathrm{db}+\mathrm{ACE} 2 \mathrm{and} \mathrm{db} / \mathrm{db}+\mathrm{GFP}$ mice exposure at $4^{\circ} \mathrm{C}$.

$\mathrm{n}=5-7 /$ each group; Data represent mean \pm SEM; ${ }^{*} p<0.05,{ }^{* *} p<0.01$ vs GFP/Vehicle group by Student's $t$-test. 
A

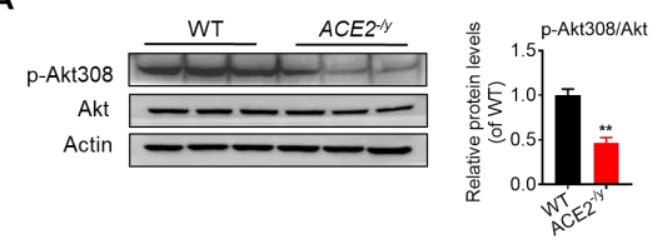

C
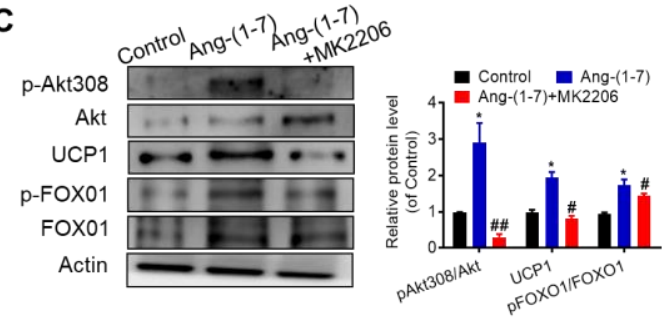

B

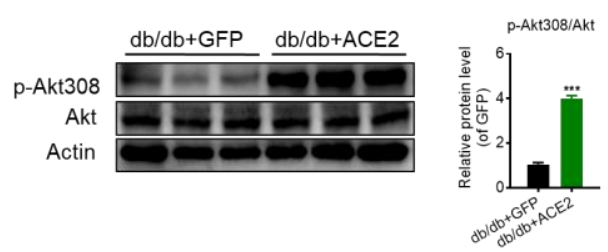

Figure 6.
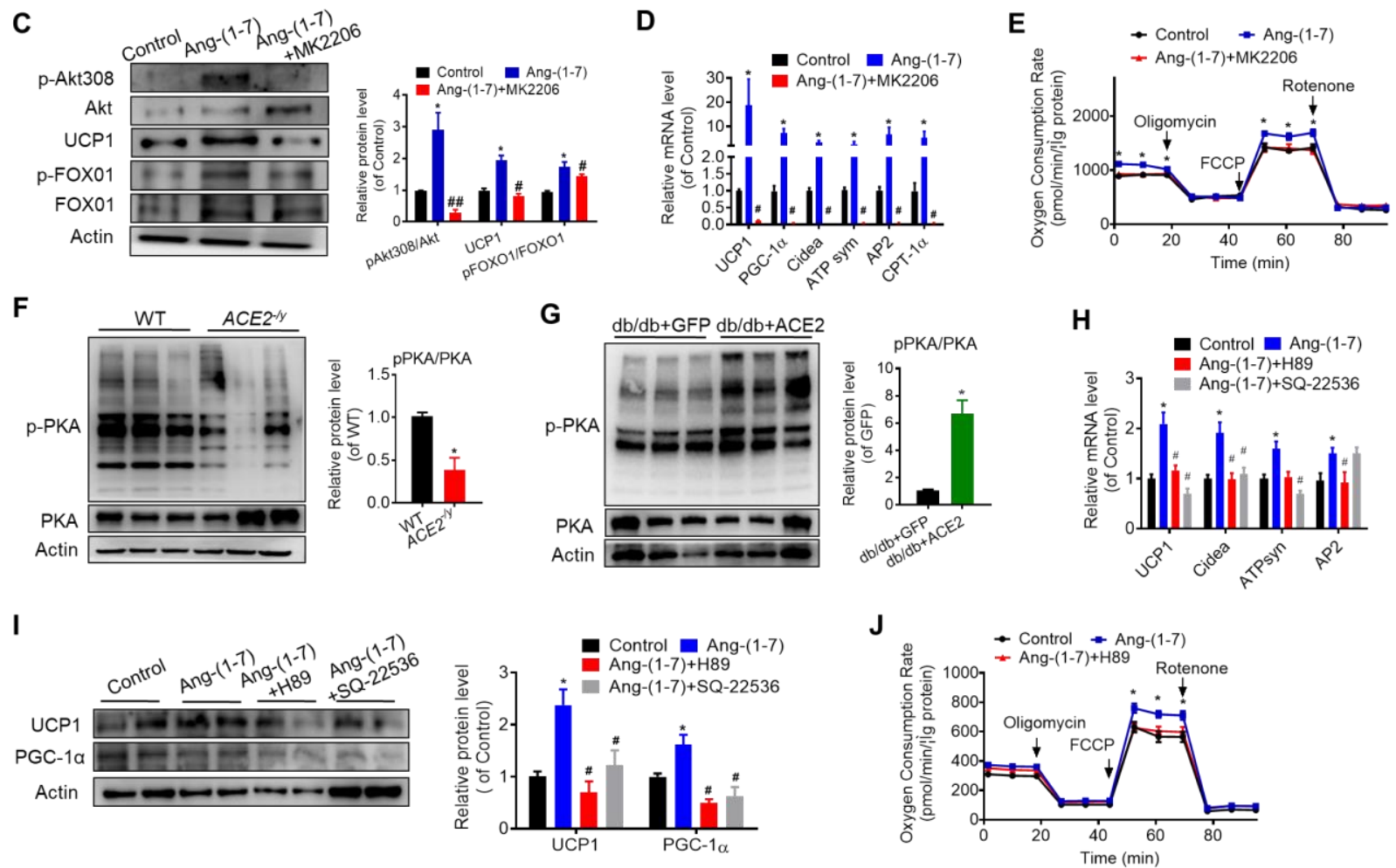

1

$\mathbf{K}$

2

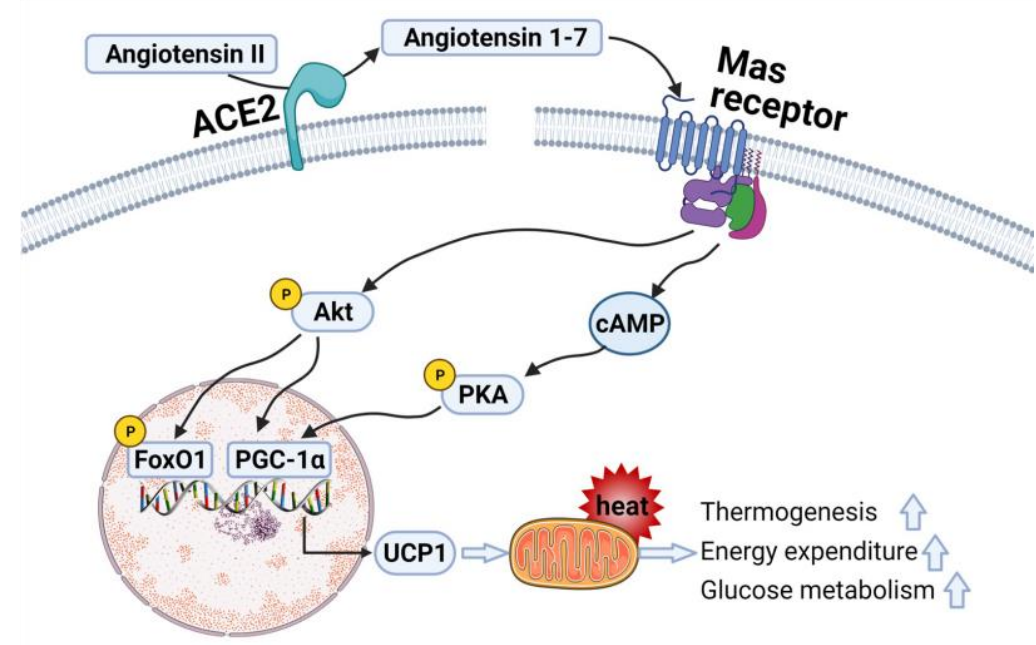
Figure 6. ACE2 pathway induces a thermogenesis program through the Akt signaling and the PKA signaling
Primary brown adipocytes were isolated, cultured, and treated with Ang- $(1-7)\left(10^{-6} \mathrm{M}\right)$ for

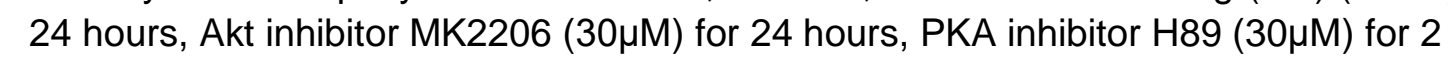
hours, or adenylylcyclase inhibitor SQ-22536 $(10 \mu \mathrm{M})$ for 24 hours. 
1 (A, B) Representative western blots showing the changes of $p$-Akt308 and Akt in BAT of

$2 \quad A_{C E 2}{ }^{-1 y}(\mathbf{A})$ and $\mathrm{db} / \mathrm{db}+\mathrm{ACE} 2$ mice $(\mathbf{B})$ exposure at $4^{\circ} \mathrm{C} . \mathrm{n}=3 /$ each group.

3 (C) Representative western blots showing the Akt, uncoupling protein-1 (UCP1) and 4 forkhead box protein 01 (FOXO1) changes. $n=3 /$ each group.

5 (D) Relative mRNA levels of thermogenic and mitochondrial genes. $n=4-6 /$ each group.

6 (E) Continuous measurement of oxygen consumption rate (OCR). Oxygen consumption 7 was performed under basal conditions, following the addition of oligomycin $(14 \mu \mathrm{M})$, the 8 pharmacological uncoupler FCCP $(10 \mu \mathrm{M})$ or the Complex III and I inhibitor antimycin A 9 and rotenone (4 $\mu \mathrm{M}$ each). $\mathrm{n}=5-7 /$ each group.

10 (F, G) Representative western blots showing the p-PKA and PKA changes in BAT of $11 \operatorname{ACE2}^{-1 y}(\mathbf{F})$ and db/db+ACE2 mice $(\mathbf{G})$ exposure at $4^{\circ} \mathrm{C} . \mathrm{n}=3$ /each group.

12 (H) Relative mRNA levels of thermogenic and mitochondrial genes. $n=4-6 /$ each group.

13 (I) Representative western blots showing the UCP1 and PGC-1 $\alpha$ changes. $n=4 /$ each 14 group.

15 (J) Continuous measurement of OCR. Oxygen consumption was performed under basal conditions, following the addition of oligomycin, the pharmacological uncoupler FCCP

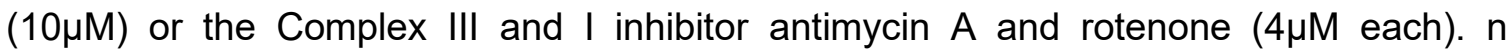
$18=5-7 /$ each group.

19 Data represent mean \pm SEM; ${ }^{*} p<0.05,{ }^{* *} p<0.01$ vs control group by Student's test. \# $p$ $20<0.05$, \#\# $p<0.01$ vs Ang-(1-7) group by Student's $t$-test.

21 (K) Mechanisms involved in ACE2 pathway activation-induced improvement of BAT 22 function.

23 The online version of this article includes the following figure supplement(s) for figure 6: Figure supplement 5. RNA-Seq analysis of primary brown adipocytes from $A C E 2^{-1 y}$ and WT mice; Ang-(1-7) regulates thermogenesis through Akt and PKA signaling in BAT.

Figure supplement 6. Ang-(1-7) regulates thermogenesis through Akt and PKA 27 signaling in BAT. 


\section{Supplementary Figures}

A

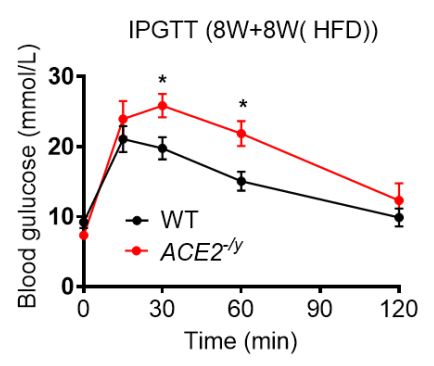

D

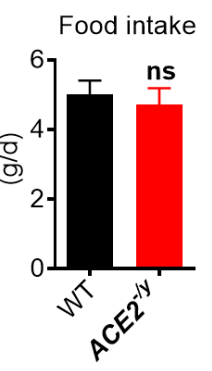

H

E
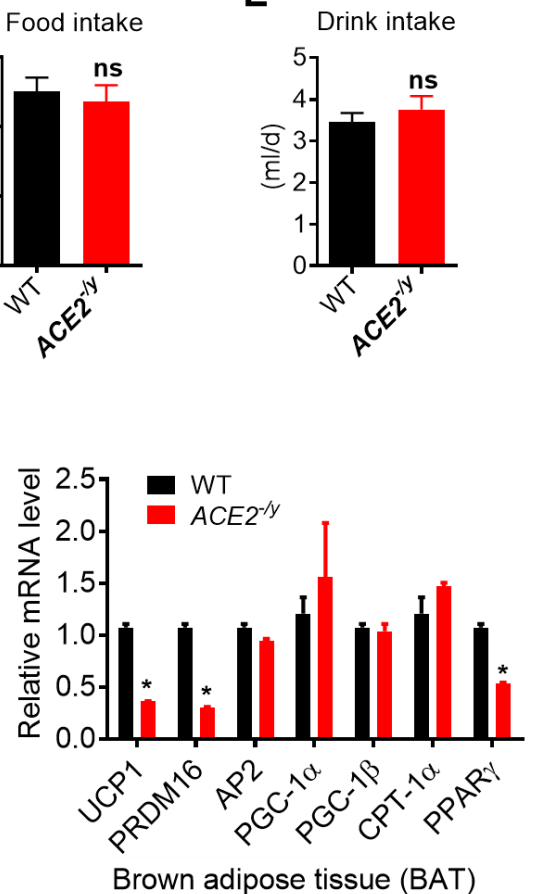

B

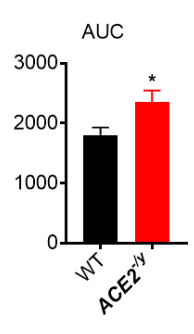

F

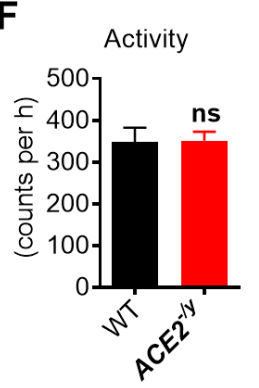

G

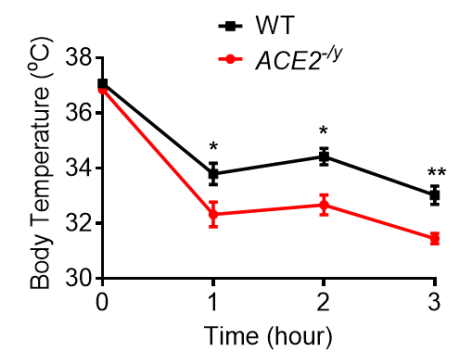

I

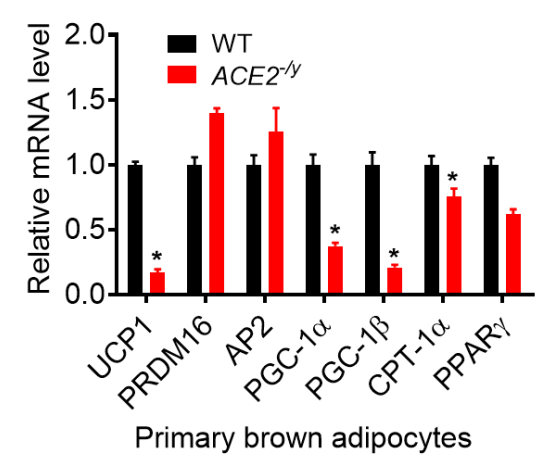

Figure supplement 1. ACE2 deficiency impairs adaptative thermogenesis by cold stimulation.

Eight-week-old male $A C E 2^{-1 y}$ mice and their WT (control) mice had an HFD for 8 weeks (ACE2-ly vs WT).

(A-C) Intraperitoneal glucose tolerance test (IPGTT), serum triglyceride and cholesterol levels in ACE2 $2^{-y}$ and WT mice.

(D-F) $24 \mathrm{~h}$ food intake, water intake and physical activity were measured in $A C E 2^{-/ y}$ and WT mice.

(G) Core body temperature at $4^{\circ} \mathrm{C}$ for the indicated lengths of time in $A C E 2^{-1 y}$ and WT mice.

(H) Relative mRNA levels of mitochondrial related genes, fatty acid oxidation related genes and transcription factors in BAT of in $A C E 2^{-1 y}$ and WT mice exposure at $4^{\circ} \mathrm{C}$.

(I) Relative mRNA levels of mitochondrial related genes, fatty acid oxidation related genes and transcription factors in primary brown adipocytes from $A C E 2^{-/ y}$ and WT mice. $\mathrm{n}=5$-7/each group; Data represent mean \pm SEM; ${ }^{*} p<0.05$, ${ }^{* *} p<0.01$ vs WT group by Student's test. 
A

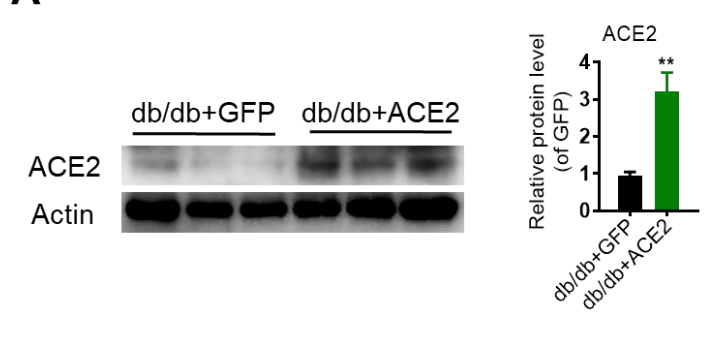

B

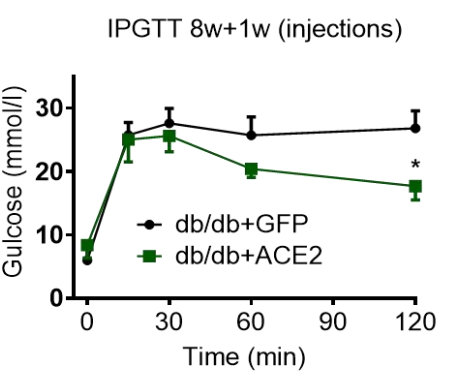

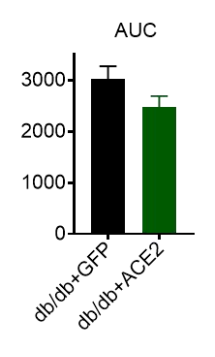

$\mathbf{F}$

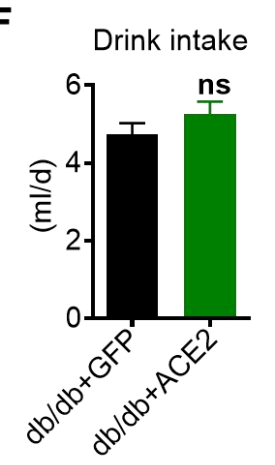

G

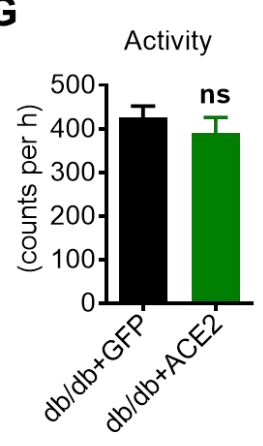

H
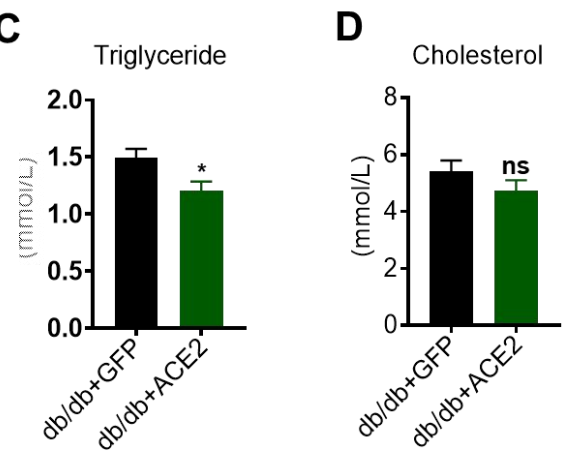

I
E

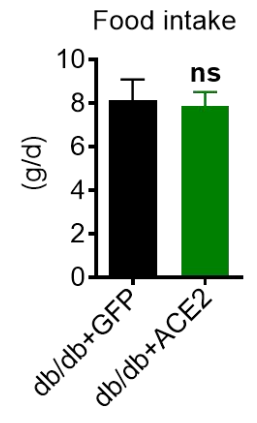

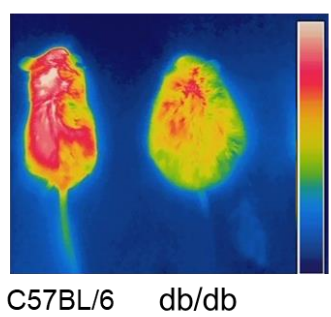

1

\section{J}

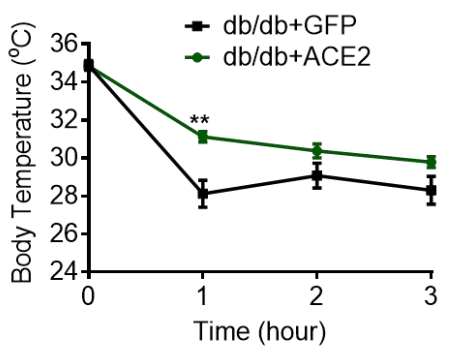

Figure supplement 2. ACE2 enhance BAT activity and whole-body energy metabolism in $\mathrm{db} / \mathrm{db}$ mice.

The ad-ACE2 and Ad-GFP treated db/db mice were used at the 6th day post-virus injection (db/db+ACE2 vs db/db+GFP).

(A) ACE2 overexpression was verified in BAT of Ad-ACE2-treated db/db mice by Western blotting.

(B-D) Intraperitoneal glucose tolerance test (IPGTT), serum triglyceride and cholesterol levels in db/db+ACE2 and db/db+GFP mice.

(E-G) $24 \mathrm{~h}$ food intake, water intake and physical activity were measured in $\mathrm{db} / \mathrm{db}+\mathrm{ACE} 2$ and $\mathrm{db} / \mathrm{db}+$ GFP mice.

(H, I) Infrared thermal images at $22^{\circ} \mathrm{C}$ and core body temperature at $4{ }^{\circ} \mathrm{C}$ for the indicated lengths of time in $\mathrm{C} 57 \mathrm{BL} / 6$ and $\mathrm{db} / \mathrm{db}$ mice. (J) Core body temperature at $4^{\circ} \mathrm{C}$ for the indicated lengths of time in $\mathrm{db} / \mathrm{db}+\mathrm{ACE} 2$ and $\mathrm{db} / \mathrm{db}+\mathrm{GFP}$ mice. $\mathrm{n}=5-7 /$ each group; Data represent mean \pm SEM; ${ }^{*} p<0.05,{ }^{* *} p<0.01$ vs WT/GFP group by Student's test. 
A

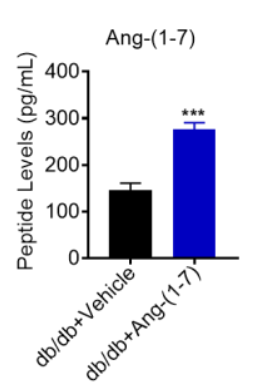

E

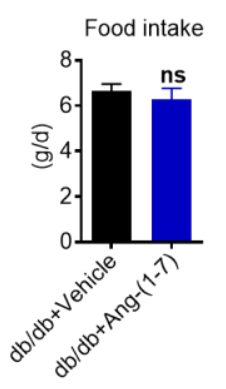

B

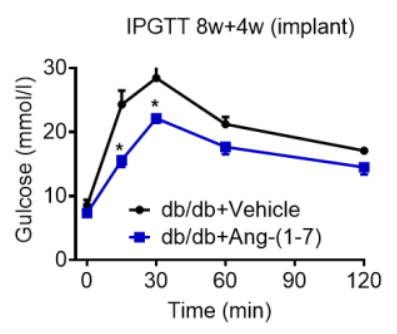

$\mathbf{F}$

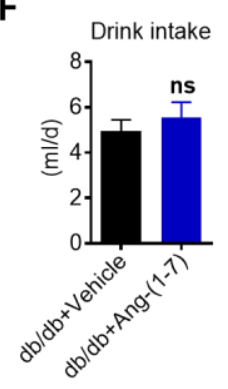

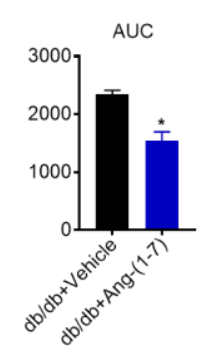

C

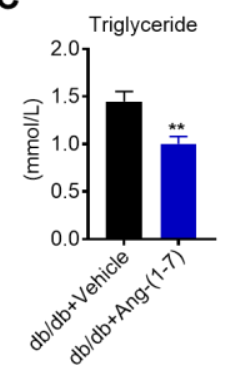

G

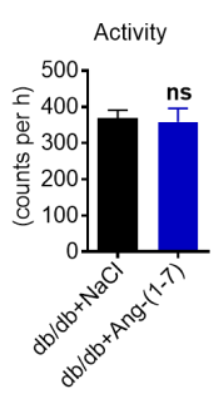

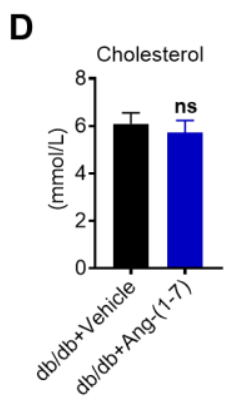

H

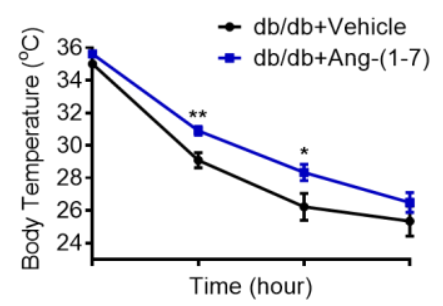

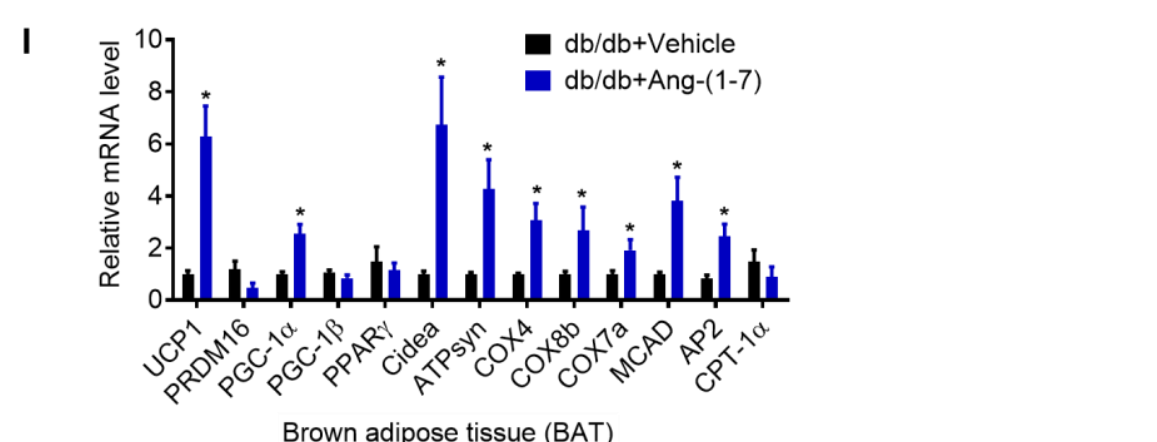

2 Figure supplement 3. Ang-(1-7) promoted thermogenesis and energetic metabolism in

3 BAT of $\mathrm{db} / \mathrm{db}$ mice during cold challenge.

$4 \mathrm{db} / \mathrm{db}$ mice were treated with Ang-(1-7) by subcutaneous infusion of Ang-(1-7) or saline using osmotic mini-pumps for 4 weeks ( $\mathrm{db} / \mathrm{db}+$ Ang-(1-7) vs $\mathrm{db} / \mathrm{db}+$ Vehicle).

(A) Serum levels of Ang-(1-7) as determined by ELISA in $\mathrm{db} / \mathrm{db}+$ Ang-(1-7) and $\mathrm{db} / \mathrm{db}+$ Vehicle mice.

(B-D) Intraperitoneal glucose tolerance test (IPGTT), serum triglyceride and cholesterol levels in $\mathrm{db} / \mathrm{db}+$ Ang-(1-7) and $\mathrm{db} / \mathrm{db}+$ Vehicle mice.

(E-G) $24 \mathrm{~h}$ food intake, water intake and physical activity were measured in $\mathrm{db} / \mathrm{db}+$ Ang-(1-7) and db/db+Vehicle mice.

(H) Core body temperature at $4^{\circ} \mathrm{C}$ for the indicated lengths of time in $\mathrm{db} / \mathrm{db}+$ Ang-(1-7) and $\mathrm{db} / \mathrm{db}+$ Vehicle mice.

(I) Relative mRNA levels of mitochondrial related genes, fatty acid oxidation related genes and transcription factors in BAT of in $\mathrm{db} / \mathrm{db}+$ Ang-(1-7) and $\mathrm{db} / \mathrm{db}+$ Vehicle mice exposure at $4^{\circ} \mathrm{C}$. $n=5-7 /$ each group; Data represent mean $\pm \mathrm{SEM}$; ${ }^{*} p<0.05,{ }^{* *} p<0.01 \mathrm{vs}$ Vehicle group by Student's test. 
bioRxiv preprint doi: https://doi.org/10.1101/2021.08.10.455823; this version posted August 10, 2021. The copyright holder for this preprint (which was not certified by peer review) is the author/funder, who has granted bioRxiv a license to display the preprint in perpetuity. It is made available under aCC-BY 4.0 International license.

4 / 7

A

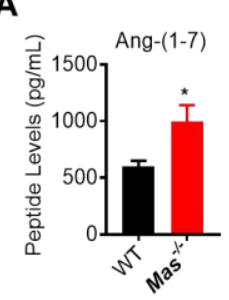

$\mathbf{F}$

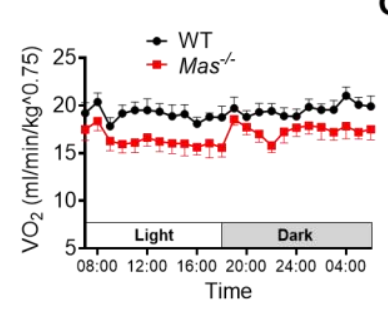

B

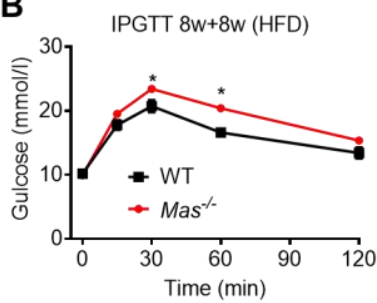

G

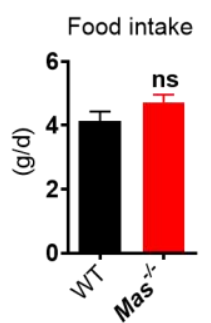

C

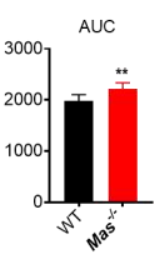

H
D

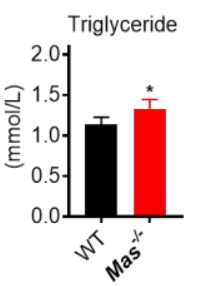

E

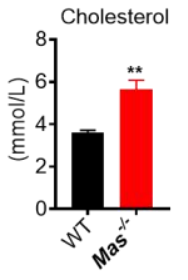

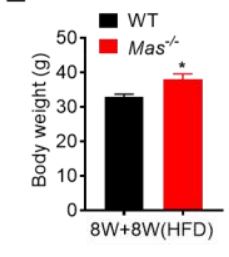

K

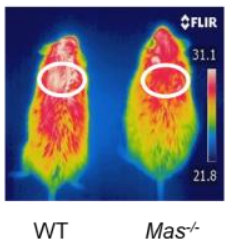

1

0

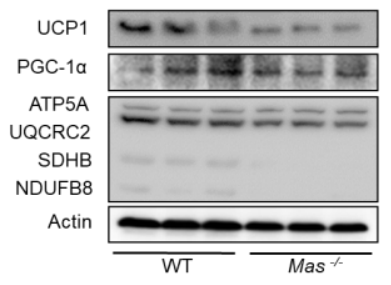

R

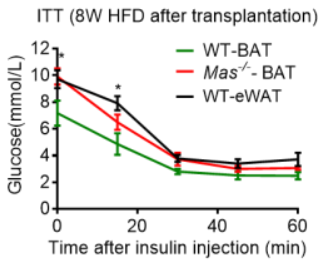

U

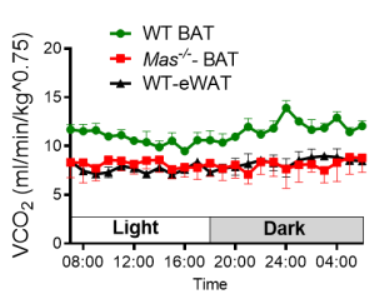

L

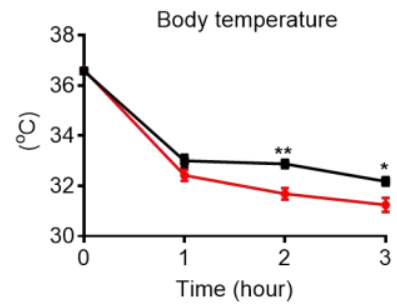

M

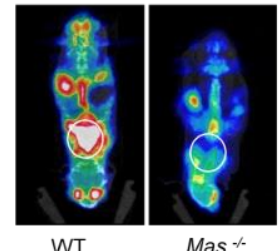

J
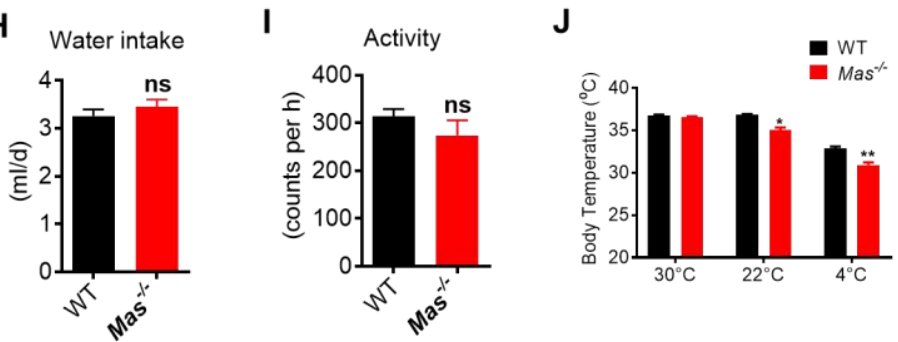

N

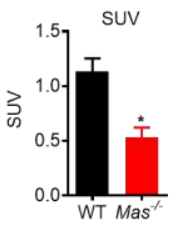

P

Q
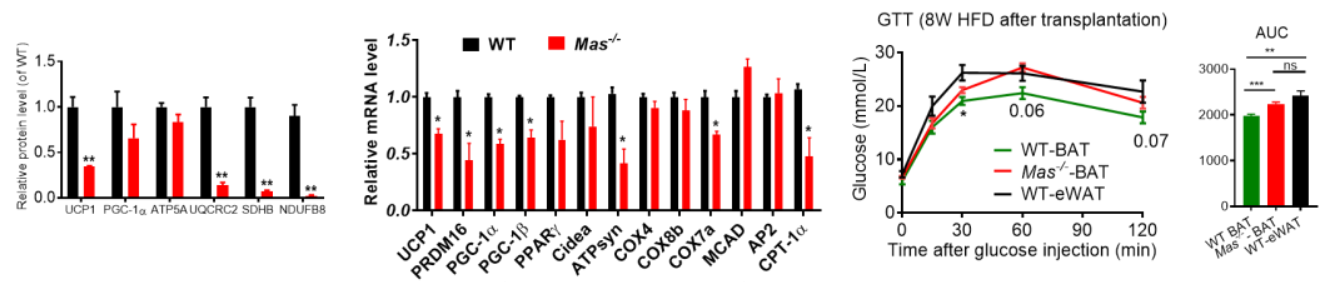

S
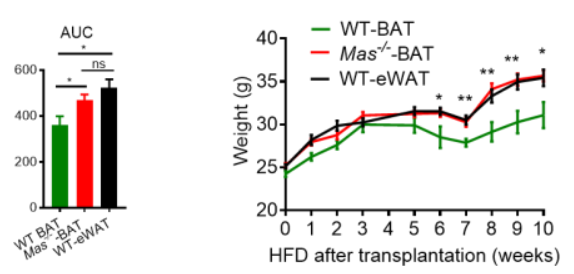

T

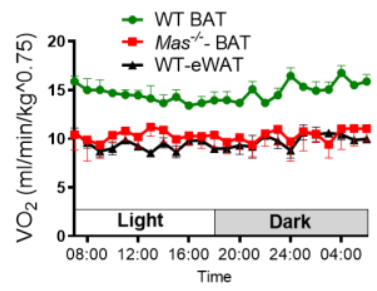

V
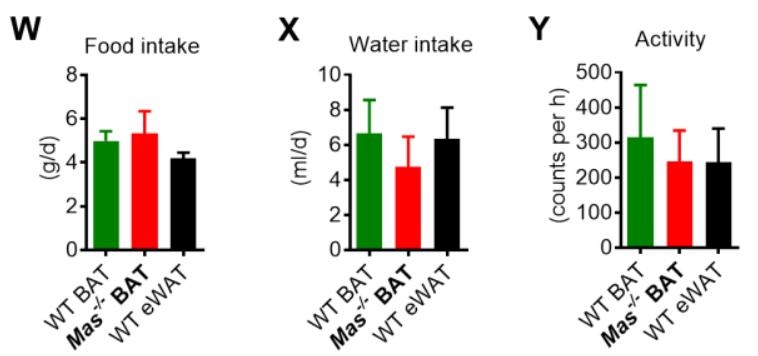
bioRxiv preprint doi: https://doi.org/10.1101/2021.08.10.455823; this version posted August 10,2021 . The copyright holder for this preprint (which was not certified by peer review) is the author/funder, who has granted bioRxiv a license to display the preprint in perpetuity. It is made available under aCC-BY 4.0 International license.

\section{$5 / 7$}

1 Figure supplement 4. Ablation of Mas impairs thermogenesis, BAT activity, and 2 energetic metabolism.

3 (A-P) Eight-week-old male $\mathrm{Mas}^{-/}$mice and their WT (control) mice had a high-fat diet 4 (HFD) for 8 weeks (Mas ${ }^{-/}$vs WT). (Q-Y) BAT of C57B/L6 recipient mice was removed 5 from the interscapular region. Then, the BAT dissected from $\mathrm{Mas}^{-/}$donor mice, was 6 subcutaneously transplanted into the dorsal interscapular region of C57B/L6 recipient 7 mice (WT+Mas $\left.{ }^{-}-\mathrm{BAT}\right)$. C57B/L6 recipient mice transplanted with C57B/L6 BAT 8 (WT+WT-BAT) and C57B/L6 epididymal white adipose tissue (eWAT) (WT+WT-eWAT) 9 were used as control. The recipient mice were then fed an HFD immediately after the transplantation and continued for 10 weeks (WT+Mas ${ }^{-1-B A T}$ vs WT+WT-BAT, WT+WT-eWAT).

(A) Serum levels of Ang-(1-7) as determined by ELISA in $\mathrm{Mas}^{-/}$and WT mice.

(B-D) Intraperitoneal glucose tolerance test (IPGTT), serum triglyceride and cholesterol levels in $\mathrm{Mas}^{-/}$and WT mice.

(E) Body weight in $\mathrm{Mas}^{-/}$and WT mice fed an HFD for 8 weeks.

(F) Energy expenditure was evaluated by measurement of oxygen consumption (VO2) over a $24 \mathrm{~h}$ period in $\mathrm{Mas}^{-/}$and WT mice.

(G-I) $24 \mathrm{~h}$ food intake, water intake and physical activity were measured in $\mathrm{Mas}^{-/}$and WT mice.

(J) Core body temperature at $30^{\circ} \mathrm{C}, 22^{\circ} \mathrm{C}$ and $4^{\circ} \mathrm{C}$ for 8 hours in $\mathrm{Mas}^{-/}$and WT mice.

(K) Infrared thermal images at $22^{\circ} \mathrm{C}$ in $\mathrm{Mas}^{-/}$and WT mice.

(L) Core body temperature at $4^{\circ} \mathrm{C}$ for the indicated lengths of time in $\mathrm{Mas}^{-/}$and WT mice.

(M) Representative PET-CT image and SUVs of $\mathrm{Mas}^{-/-}$and WT mice.

(N) Representative H\&E staining and UCP1 immunostaining from BAT sections of $\mathrm{Mas}^{-1-}$ and WT mice exposure at $4^{\circ} \mathrm{C}$.

(O) Representative western blots showing the changes of key proteins in BAT of Mas $^{-1}$ and WT mice exposure at $4^{\circ} \mathrm{C}$.

(P) Relative mRNA levels of mitochondrial related genes, fatty acid oxidation related genes and transcription factors in BAT of in $\mathrm{Mas}^{-/}$and WT mice exposure at $4^{\circ} \mathrm{C}$.

(Q) Intraperitoneal glucose tolerance test (GTT) and the average area under the curve (AUC) in WT+Mas ${ }^{-1}$-BAT, WT+WT-BAT and WT+WT-eWAT mice fed an HFD for 8 weeks after transplantation.

(R) Insulin tolerance test (ITT) and AUC in WT+Mas ${ }^{--B A T, ~ W T+W T-B A T ~ a n d ~}$ WT+WT-eWAT mice fed an HFD for 8 weeks after transplantation.

(S) Body weight time course in WT+Mas ${ }^{-}-\mathrm{BAT}$, WT+WT-BAT and WT+WT-eWAT mice fed an HFD over 10 weeks after transplantation.

(T-V) Energy expenditure was evaluated by measurement of oxygen consumption $\left(\mathrm{VO}_{2}\right)$ $(\mathbf{T})$, of carbon dioxide release $\left(\mathrm{VCO}_{2}\right)(\mathbf{U})$ and of energy expenditure $(\mathrm{EE})(\mathbf{V})$ over a $24 \mathrm{~h}$ period in WT+Mas ${ }^{-}-$BAT, WT+WT-BAT and WT+WT-eWAT mice.

(W-Y) $24 \mathrm{~h}$ food intake, water intake and physical activity were measured in WT+Mas ${ }^{-1}$-BAT, WT+WT-BAT and WT+WT-eWAT mice fed an HFD for 8 weeks after transplantation. $\mathrm{n}=5-7 /$ each group; Data represent mean $\pm \mathrm{SEM} ;{ }^{*} p<0.05,{ }^{* *} p<0.01$ vs WT/ WT+WT-BAT group by Student's $t$-test. 
A

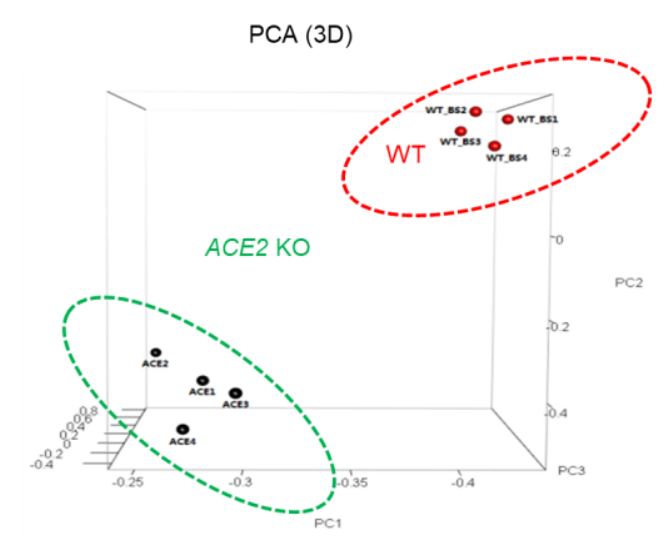

C

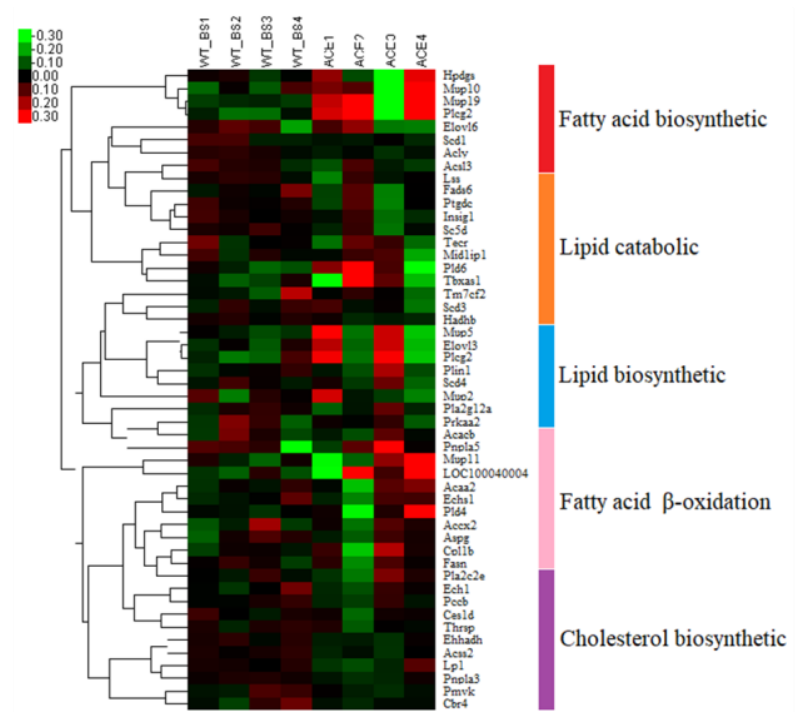

B

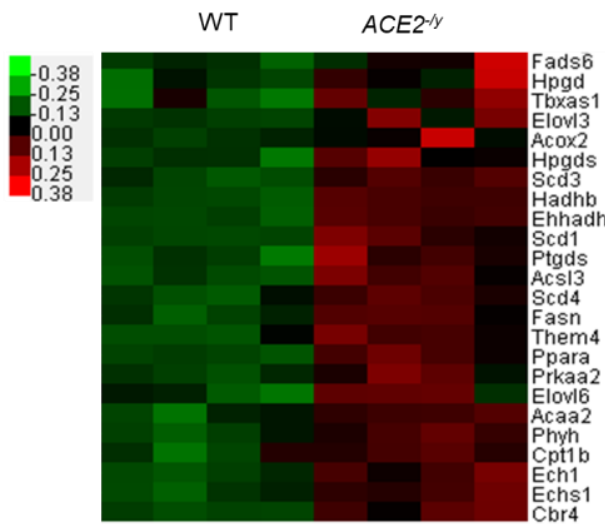

Fatty acid metabolic

D
1

Figure supplement 5. RNA-Seq analysis of primary brown adipocytes from $A C E 2^{-y}$ and WT mice.

(A) 3D-PCA analysis represent the deviation of 4 replication within ACE2-1y (ACE2 1-4) and WT mice (WT_BS 1-4).

(B) Heat map representation of the differentially expressed genes in $A C E 2^{-1 y}$ and WT mice. Gene expression is coded in color scale (-2 to 2). Red or green indicates expression levels above or below the median, respectively. The magnitude of deviation from the median is represented by color saturation.

(C) Heat map of selected genes associated with fatty acid biosynthetic, lipid catabolic, lipid biosynthetic, fatty acid beta-oxidation and cholesterol biosynthetic, which derived from GO analysis results.

(D) Table for the expression of Akt signal pathways related genes. 
A

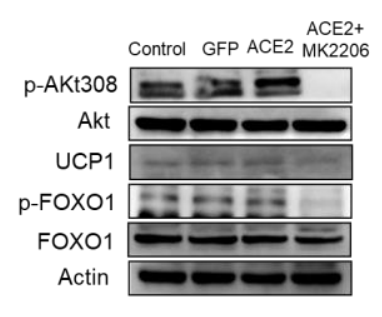

B

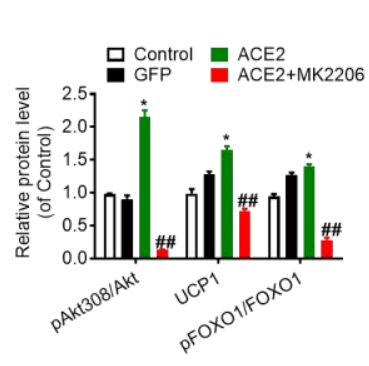

D

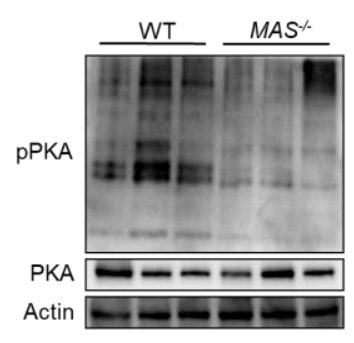

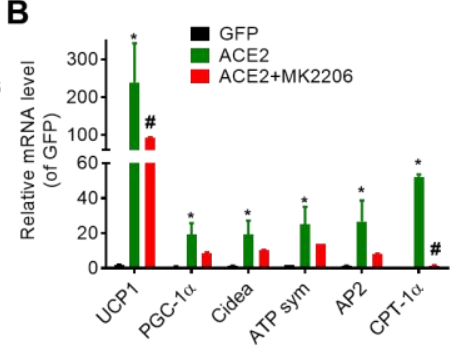

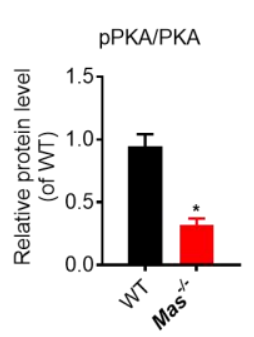

E

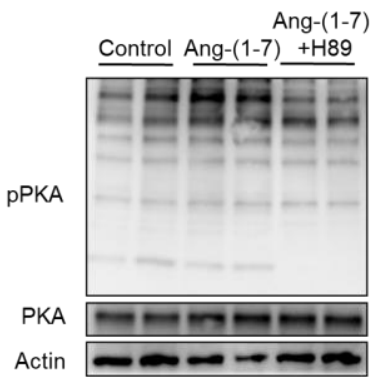

C

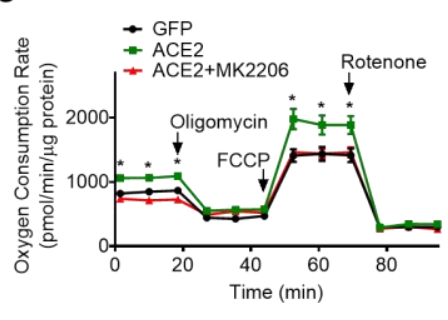

1

Figure supplement 6. Ang-(1-7) regulates thermogenesis through Akt and PKA signaling in BAT.

Primary brown adipocytes were isolated, cultured, infected with ACE2 adenovirus,

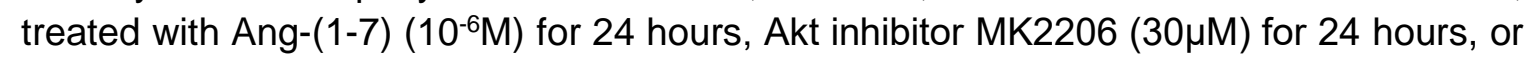
PKA inhibitor $\mathrm{H} 89$ (30 $\mathrm{MM})$ for 2 hours.

(A) Representative western blots showing the Akt, UCP1 and FoxO1 changes in primary brown adipocytes overexpressing ACE2 at day 7 and treated with MK2206. $n=3 /$ each group.

(B) Relative mRNA levels of thermogenic and mitochondrial genes. $n=4-6 /$ each group.

(C) Continuous measurement of OCR. Oxygen consumption was performed under basal conditions, following the addition of oligomycin $(14 \mu \mathrm{M})$, the pharmacological uncoupler FCCP $(10 \mu \mathrm{M})$ or the Complex III and I inhibitor antimycin A and rotenone ( $4 \mu \mathrm{M}$ each). $\mathrm{n}$ $=4-7 /$ each group.

(D) Representative western blots showing the p-PKA and PKA changes in BAT of Mas $^{-/}$ mice exposure at $4^{\circ} \mathrm{C} . \mathrm{n}=3$ /each group.

(E) Representative western blots showing the p-PKA and PKA changes in Ang-(1-7) and $\mathrm{H} 89$ treated primary brown adipocytes. $n=4$ /each group. Data represent mean $\pm \mathrm{SEM} ;{ }^{*} p$ $<0.05,{ }^{* *} p<0.01$ vs GFP/WT/control group by Student's test. $\# p<0.05, \# \# p<0.01$ vs ACE2/Ang-(1-7) group by Student's $t$-test. 